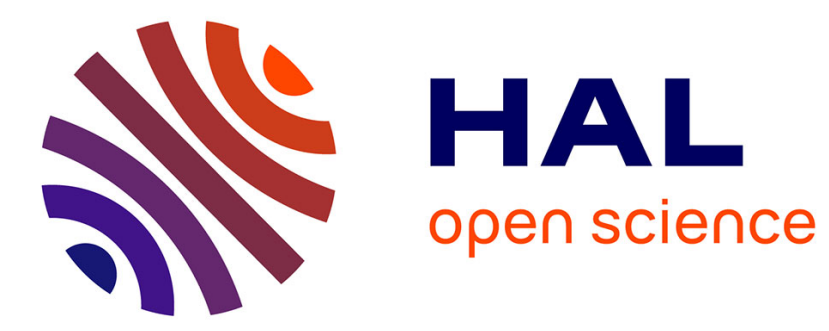

\title{
Liouville type theorems, monotonicity results and a priori bounds for positive solutions of elliptic systems
}

Djairo G. de Figueiredo, Boyan Sirakov

\section{To cite this version:}

Djairo G. de Figueiredo, Boyan Sirakov. Liouville type theorems, monotonicity results and a priori bounds for positive solutions of elliptic systems. Mathematische Annalen, 2005, 333, pp.231-260. hal-00004766

\section{HAL Id: hal-00004766 https://hal.science/hal-00004766}

Submitted on 20 Apr 2005

HAL is a multi-disciplinary open access archive for the deposit and dissemination of scientific research documents, whether they are published or not. The documents may come from teaching and research institutions in France or abroad, or from public or private research centers.
L'archive ouverte pluridisciplinaire HAL, est destinée au dépôt et à la diffusion de documents scientifiques de niveau recherche, publiés ou non, émanant des établissements d'enseignement et de recherche français ou étrangers, des laboratoires publics ou privés. 


\title{
Liouville type theorems, monotonicity results and a priori bounds for positive solutions of elliptic systems
}

\author{
Djairo G. de FIGUEIREDO \\ IMECC-UNICAMP \\ Caixa Postal 6065 \\ Campinas, S.P. \\ BRAZIL \\ Boyan SIRAKOV \\ Laboratoire MODALX, UFR SEGMI \\ Université Paris 10, Bâtiment G \\ 92001 Nanterre Cedex \\ FRANCE
}

\section{Introduction}

This paper is a contribution to the study of boundary value problems for systems of elliptic partial differential equations of the form

$$
\left\{\begin{array}{rlr}
-\Delta u_{1}=f\left(x, u_{1}, u_{2}\right) & \text { in } \quad \Omega \\
-\Delta u_{2}=g\left(x, u_{1}, u_{2}\right) & \text { in } \quad \Omega \\
u_{1}=u_{2}=0 & \text { on } \partial \Omega
\end{array}\right.
$$

where $u_{1}, u_{2}$ are real-valued functions defined on a smooth bounded domain $\Omega$ in $\mathbb{R}^{N}, N \geq 3$, and $f$ and $g$ are Hölder continuous functions defined in $\bar{\Omega} \times \mathbb{R} \times \mathbb{R}$.

This type of systems has been extensively studied during the last two decades - see for example the survey paper [19] and the references therein. One of the important questions is the existence of a priori bounds for positive smooth solutions of these systems.

It is well known that the existence of a priori bounds depends on the growth of the functions $f$ and $g$ as $u_{1}$ and $u_{2}$ go to infinity. In view of what is known for scalar equations, one expects that some polynomial (subcritical) growth is to be required. In fact such a restriction comes from the Sobolev imbedding theorems in dimension $N \geq 3$. It is also known that a priori bounds are particularly interesting when superlinear equations are considered. In fact, it is classical (see [27], [4], [3], [34]) that establishing a priori bounds for a scalar equation permits, through use of Krasnoselskii's index 
theory, to obtain existence results for such an equation. Very recently it was shown that systems have an analogous property and definitions of superlinearity for nonvariational systems of two equations were given in [2] ; see also [19] and [40] for applications of index theory to some special types of systems. More general results can be found in [37] where, in addition, systems of many equations are considered.

The simplest case of systems of type (1) — which is the only case in which a priori bounds have been studied up to now - is when the leading parts of $f$ and $g$ involve just pure powers of $u_{1}$ and $u_{2}$. More precisely, when $f$ and $g$ are such that $(1)$ can be written in the form

$$
\left\{\begin{array}{l}
-\Delta u_{1}=a(x) u_{1}^{\alpha_{11}}+b(x) u_{2}^{\alpha_{12}}+h_{1}\left(x, u_{1}, u_{2}\right) \\
-\Delta u_{2}=c(x) u_{1}^{\alpha_{21}}+d(x) u_{2}^{\alpha_{22}}+h_{2}\left(x, u_{1}, u_{2}\right)
\end{array}\right.
$$

where the exponents $\alpha_{i j}$ are nonnegative real numbers, $a(x), b(x), c(x), d(x)$ are nonnegative continuous functions on $\bar{\Omega}$, and $h_{1}, h_{2}$ are locally bounded functions such that uniformly in $x \in \Omega$

$$
\left\{\begin{array}{l}
\lim _{\left|\left(u_{1}, u_{2}\right)\right| \rightarrow \infty}\left(a(x) u_{1}^{\alpha_{11}}+b(x) u_{2}^{\alpha_{12}}\right)^{-1}\left|h_{1}\left(x, u_{1}, u_{2}\right)\right|=0 \\
\lim _{\left|\left(u_{1}, u_{2}\right)\right| \rightarrow \infty}\left(c(x) u_{1}^{\alpha_{21}}+d(x) u_{2}^{\alpha_{22}}\right)^{-1}\left|h_{2}\left(x, u_{1}, u_{2}\right)\right|=0
\end{array}\right.
$$

The method used here in order to obtain the a priori bounds, the so-called blow-up method, was introduced in [24] to treat the scalar case. The use of this method to treat systems like the one in (2) was first done in [38], and then in [33], [8], [19], [40]. Let us note that the blow-up method itself depends on results of nonexistence of positive solutions of equations and systems in the whole space or in a half-space. Such results are usually referred to as Liouville type theorems - see Section 2.

Our main result, Theorem 1.1, unifies and extends the previous results on a priori bounds for (2). In addition, it allows more general nonlinearities in systems of type (1), namely mixed powers of $u_{1}$ and $u_{2}$ in the principal part of the nonlinearities $f$ and $g$.

Specifically, our results will concern the following system

$$
\left\{\begin{array}{l}
-\Delta u_{1}=a(x) u_{1}^{\alpha_{11}}+b(x) u_{2}^{\alpha_{12}}+f_{1}(x) u_{1}^{\gamma_{11}} u_{2}^{\gamma_{12}}+h_{1}\left(x, u_{1}, u_{2}\right) \\
-\Delta u_{2}=c(x) u_{1}^{\alpha_{21}}+d(x) u_{2}^{\alpha_{22}}+f_{2}(x) u_{1}^{\gamma_{21}} u_{2}^{\gamma_{22}}+h_{2}\left(x, u_{1}, u_{2}\right)
\end{array}\right.
$$

where we keep all the hypotheses made before for the system (2).

As for new hypotheses for system (4), we suppose that the continuous functions $f_{1}$ and $f_{2}$ are nonnegative in $\Omega$, so (2) is a particular case of (4), and 
$\left(\gamma_{\mathbf{1}}\right) \quad$ we have $0 \leq \gamma_{i j} \leq \alpha_{i j}, i, j=1,2$,

$$
\frac{\gamma_{11}}{\alpha_{11}}+\frac{\gamma_{12}}{\alpha_{12}}=1 \quad \text { and } \quad \frac{\gamma_{21}}{\alpha_{21}}+\frac{\gamma_{22}}{\alpha_{22}}=1
$$

Hypothesis (5) means that the powers $\gamma_{i j}$ are such that the terms with coefficients $f_{i}$ in (4) are exactly of the order of the principal parts in (2) and cannot be included in the functions $h_{1}, h_{2}$, via Young's inequality.

We make the following superlinearity assumptions on the exponents $\alpha_{i j}$ :

$$
\text { either } \quad \alpha_{11}>1, \quad \text { or } \quad \alpha_{22}>1, \quad \text { or } \quad \alpha_{12} \alpha_{21}>1 \text {. }
$$

We remark here that $\alpha_{i i}>1$ means the Emden-Fowler equation $-\Delta u_{i}=u_{i}^{\alpha_{i i}}$ is superlinear, while the third inequality in (6) has long been used as a notion of superlinearity for the Lane-Emden system

$$
\left\{\begin{array}{l}
-\Delta u_{1}=u_{2}^{\alpha_{12}} \\
-\Delta u_{2}=u_{1}^{\alpha_{21}}
\end{array}\right.
$$

Further, we have to specify the maximal growth of $f$ and $g$ in (1) that we can allow. This can best be done by using the following geometric construction. We denote $\vec{\beta}=\left(\beta_{1}, \beta_{2}\right) \in \mathbb{R}^{2}$, and introduce the following lines (see Figure 1 in Section 2)

$$
\begin{aligned}
& l_{1}=\left\{\vec{\beta} \mid \beta_{1}+2-\beta_{1} \alpha_{11}=0\right\}, \quad l_{2}=\left\{\vec{\beta} \mid \beta_{2}+2-\beta_{2} \alpha_{22}=0\right\}, \\
& l_{3}=\left\{\vec{\beta} \mid \beta_{1}+2-\beta_{2} \alpha_{12}=0\right\}, \quad l_{4}=\left\{\vec{\beta} \mid \beta_{2}+2-\beta_{1} \alpha_{21}=0\right\} .
\end{aligned}
$$

A thorough explanation of how these lines appear will be given in the next section, when we expound the blow-up method. We just note here that if we set $\left(\beta_{1}^{\prime}, \beta_{2}^{\prime}\right)=l_{1} \cap l_{2}$ (in case $\alpha_{11}, \alpha_{22}>1$ ) and $\left(\beta_{1}^{\prime \prime}, \beta_{2}^{\prime \prime}\right)=l_{3} \cap l_{4}$ (in case $\left.\alpha_{12} \alpha_{21}>1\right)$, then the Emden-Fowler equations $-\Delta u_{i}=u_{i}^{\alpha_{i i}}$ are subcritical provided $\beta_{i}^{\prime}>\frac{N-2}{2}$, while system (7) is subcritical provided $\beta_{1}^{\prime \prime}+\beta_{2}^{\prime \prime}>N-2$. Actually, the last condition is equivalent to saying that the exponents $\alpha_{12}, \alpha_{21}$ in (7) are under the so-called "critical hyperbola" — a widely used notion of criticality for (7), introduced in [16] and [26].

We consider points $\vec{\beta} \geq 0$ which are to the left of or on $l_{1}$, below or on $l_{2}$ (note that both $l_{1}$ and $l_{2}$ can be empty, and then they introduce no restriction), below or on $l_{3}$, and above or on $l_{4}$. We call these points admissible (this notion will be completely understood in the next section). So given a system of type (2) the set of admissible points is automatically defined. 
We divide the systems of type (2) into three classes, determined by the exponents $\alpha_{i j}$. In each case we make a choice of $\left(\beta_{1}, \beta_{2}\right)$ - which we shall use to state our theorem - and make some assumptions on the coefficients of the system.

Case A. The intersection of $l_{1}$ and $l_{2}$ is admissible. Then we set $\left(\beta_{1}, \beta_{2}\right)=$ $l_{1} \cap l_{2}$. In this case we shall assume that the functions $a(x)$ and $d(x)$ are bounded below on $\bar{\Omega}$ by a positive constant.

Case B. The intersection of $l_{3}$ and $l_{4}$ is admissible. For this type of systems we take $\left(\beta_{1}, \beta_{2}\right)=l_{3} \cap l_{4}$. In this case we shall assume that the functions $b(x)$ and $c(x)$ are bounded below on $\bar{\Omega}$ by a positive constant. Further, in case $\mathrm{B}$ we have to assume in addition that $\alpha_{12}>1$ and $\alpha_{21}>1$ - see Remark 2 below.

Case C. None of $l_{1} \cap l_{2}$ and $l_{3} \cap l_{4}$ is admissible. Then either $l_{1} \cap l_{3}$ or $l_{2} \cap l_{4}$ is admissible and we take this intersection point to be our $\left(\beta_{1}, \beta_{2}\right)$. In this case we shall assume that the function $b(x)(\operatorname{resp} . c(x))$ is bounded below on $\bar{\Omega}$ by a positive constant.

We stress that nothing prevents $\left(\beta_{1}, \beta_{2}\right)$ from being the intersection of more than two lines. Note that a system can be simultaneously of type $\mathrm{A}$ and $\mathrm{B}$, but type $\mathrm{C}$ is exclusive of the other two types.

In the special situation in Case $B$ when $\beta$ is the intersection of more than two lines we shall need in our arguments below a further technical assumption on the $\gamma_{i j}$ (we believe this hypothesis can be removed) :

$$
\left(\gamma_{\mathbf{2}}\right) \gamma_{11}, \gamma_{12} \geq 1 \text {, if } \vec{\beta}=l_{1} \cap l_{3} \cap l_{4}, \quad ; \quad \gamma_{21}, \gamma_{22} \geq 1 \text {, if } \vec{\beta}=l_{2} \cap l_{3} \cap l_{4} .
$$

Theorem 1.1 Assume that system (4) satisfies the conditions stated above, and that the pair $\left(\beta_{1}, \beta_{2}\right)$ which corresponds to the type of the system $(A, B$ or $C$ ) satisfies the condition

$$
\min \left\{\beta_{1}, \beta_{2}\right\}>\frac{N-2}{2} .
$$

Then system (4) admits a priori estimates, that is, each couple of positive classical solutions of (4) is bounded in the $L^{\infty}$-norm by a constant which depends only on $L^{\infty}$-bounds for the coefficients of the system and on the domain.

Remark 1. In Case $\mathrm{C}$ it is actually sufficient to suppose that $\beta_{1}>\frac{N-2}{2}$ if $l_{1} \cap l_{3}$ is admissible, and that $\beta_{2}>\frac{N-2}{2}$ if $l_{2} \cap l_{4}$ is admissible. 
Remark 2. Note the additional assumption we have made in Case B - that both exponents $\alpha_{12}$ and $\alpha_{21}$ are greater than 1 . This is due to the problem (7) in $\mathbb{R}_{+}^{N}$. We do not actually know of any Liouville-type result on this problem in a half-space, which does not require this hypothesis. See the detailed discussion at the end of the next section.

Remark 3. In [19] the first author presented the blow-up procedure for systems of type (2) and observed that there exist two special types of these systems (called weakly coupled and strongly coupled in [19]), contained in Cases $\mathrm{A}$ and $\mathrm{B}$ respectively, for which the blow-up procedure leads to Liouville type results for the equation $-\Delta u=u^{p}$ or for the Lane-Emden system (7).

In [40] Zou considered system (2) and showed that it admits a priori estimates under the supplementary assumptions that all exponents $\alpha_{i j}>1$, that both coordinates of the point $l_{1} \cap l_{2}$ are larger than $\frac{N-2}{2}$, that both coordinates of the point $l_{3} \cap l_{4}$ are larger than $\frac{N-2}{2}$ (or one of them is larger than $N-2$ ), and that the point $l_{3} \cap l_{4}$ lies neither on $l_{1}$ nor on $l_{2}$. Note that the hypotheses $\alpha_{12}>1, \alpha_{21}>1$ are not stated in [40] but they are actually used in the proofs of the results, because of the previous remark.

Remark 4. The question of non-existence of positive bounded solutions of (7) in $\mathbb{R}^{N}$ was completely solved when $N=3$ by Serrin and Zou, [36]. So, when $N=3$ one can get more precise results than Theorem 1.1 - see [40], Theorems 1.1, 2.1, and 3.1 for system (2).

As noted before, implementing the blow-up method depends on availability of Liouville type results in $\mathbb{R}^{N}$ or in a half-space. In a number of situations that we are led to consider the results available in the literature (see Section 2) are not sufficient, so we had to prove Liouville type theorems for systems. Let us stress that our paper differs in that respect from all previous works on a priori bounds for systems, where the conditions on the system were actually chosen so that the limit process in the blow-up method (see Section 2 for details) leads to known Liouville type theorems.

We prove nonexistence results in a half-space for limit systems of (4) by showing that whenever such a system does not admit bounded solutions in $\mathbb{R}^{N-1}$ then it does not possess bounded solutions in any half-space of $\mathbb{R}^{N}$ either (so actually it is enough to prove the required Liouville results in the whole space). We prove this fact by using a monotonicity result for autonomous systems in a half-space. Both these theorems - of clear independent interest - hold under some supplementary assumptions that we list next. 
Suppose we have an autonomous system of the type

$$
\left\{\begin{array}{l}
\Delta u_{1}+f_{1}\left(u_{1}, u_{2}\right)=0 \\
\Delta u_{2}+f_{2}\left(u_{1}, u_{2}\right)=0
\end{array}\right.
$$

where $f_{i} \in C^{1}\left(\mathbb{R}^{2}\right), i=1,2$, and

$$
\frac{\partial f_{i}}{\partial u_{j}}(\vec{u}) \geq 0 \quad \text { for all } i \neq j, \vec{u} \in \mathbb{R}^{2} .
$$

Systems satisfying the last property are usually referred to as cooperative (or quasi-monotone). We shall suppose in addition that we can write

$$
f_{1}\left(u_{1}, u_{2}\right)=u_{2}^{p}+g_{1}\left(u_{1}, u_{2}\right) u_{1}, \quad f_{2}\left(u_{1}, u_{2}\right)=u_{1}^{q}+g_{2}\left(u_{1}, u_{2}\right) u_{2},
$$

for some $p, q>1$ and some continuous functions $g_{1}, g_{2}$, which have polynomial growth in $u_{1}, u_{2}$.

Here are the precise statements of the results.

Theorem 1.2 Suppose we have a nontrivial nonnegative bounded classical solution $\left(u_{1}, u_{2}\right)$ of system (9) in $\mathbb{R}_{+}^{N}=\left\{x \in \mathbb{R}^{N} \mid x_{N}>0\right\}$, such that $u_{1}=u_{2}=0$ on $\partial \mathbb{R}_{+}^{N}$. Suppose (10) and (11) are satisfied. Then

$$
\frac{\partial u_{i}}{\partial x_{N}}>0 \quad \text { in } \mathbb{R}_{+}^{N}, \quad i=1,2
$$

Theorem 1.3 Suppose we have a system of type (9) which satisfies (10) and (11). If problem (9) with Dirichlet boundary condition has a nontrivial nonnegative bounded solution in $\mathbb{R}_{+}^{N}$, then the same problem has a positive solution in $\mathbb{R}^{N-1}$ (the limit as $x_{N} \rightarrow \infty$ of the solution in $\mathbb{R}_{+}^{N}$ ).

We note that Dancer [17] obtained property (12) for the scalar equation $-\Delta u=f(u)$ provided that either $f(0)>0$ or both $f(0)=0$ and $f^{\prime}(0) \geq 0$. Dancer's result was proved to hold for unbounded solutions and globally Lipschitz continuous $f$ with $f(0) \geq 0$ by Berestycki, Caffarelli and Nirenberg ([6]). In this paper the authors also showed that positive solutions of $-\Delta u=$ $f(u)$ are functions of $x_{N}$ only, provided $f(\sup u) \leq 0$. They obtained even stronger results in dimensions 2 and 3.

The proof of Theorem 1.2 is based on the moving planes method and has two main ingredients. First, we use a maximum principle for cooperative systems in unbounded narrow domains, which follows from a result on scalar equations by Cabre [13]. Second, we make use of a recent Harnack type inequality for nonlinear systems obtained in [12]. By using some ideas from [12] we prove the following Harnack inequality for system (9). 
Theorem 1.4 Let $\left(u_{1}, u_{2}\right)$ be a positive solution of (9) in some domain $G$ and suppose (10) and (11) hold. Suppose $K$ is a compact set properly included in $G$ and

$$
\max \left\{\inf _{x \in K} u_{1}, \inf _{x \in K} u_{2}\right\} \leq 1, \quad \max \left\{\sup _{x \in G} u_{1}, \sup _{x \in G} u_{2}\right\} \leq M .
$$

Then

$$
\sup _{x \in K} \max \left\{u_{1}, u_{2}\right\} \leq C \min \left\{\left(\inf _{x \in K} u_{1}\right)^{\frac{1}{p}},\left(\inf _{x \in K} u_{2}\right)^{\frac{1}{q}}\right\} .
$$

where $C$ depends only on $N$ and $M$.

This inequality permits to us to use a technique inspired by the proof of a symmetry result for scalar equations in cylinders in [6]. However, contrary to [6], we avoid using boundary Harnack inequalities. A supplementary difficulty in the argument stems from the fact that we have to use a Harnack inequality on a sequence of systems in which the coupling degenerates.

Finally, we prove some Liouville type results for systems in the whole space by, on one hand, extending to general systems a recent monotonicity result by Busca and Manasevich [10], who obtained a Liouville type theorem for the Lane-Emden system (7), and, on the other hand, by noticing an identity between exponents appearing after a blow-up change of variables and after the passage to polar coordinates used in [10]. For instance, we obtain the following result.

Theorem 1.5 The system

$$
\left\{\begin{array}{l}
\Delta u_{1}+u_{1}^{\alpha_{1}}+u_{2}^{\alpha_{1} \frac{\alpha_{2}-1}{\alpha_{1}-1}}=0 \\
\Delta u_{2}+u_{1}^{\alpha_{2} \frac{\alpha_{1}-1}{\alpha_{2}-1}}+u_{2}^{\alpha_{2}}=0,
\end{array}\right.
$$

does not have bounded positive classical solutions in $\mathbb{R}^{N}$, provided

$$
1<\alpha_{1}, \alpha_{2}<\frac{N+2}{N-2} .
$$

The paper is organized as follows. In Section 2 we give some preliminary results and state the known Liouville-type results for scalar equations and Lane-Emden systems in $\mathbb{R}^{N}$ or $\mathbb{R}_{+}^{N}$. In Section 3 we prove Theorem 1.2 and Theorem 1.3. Finally, in Section 4 we prove Theorem 1.5 and Theorem 1.1. 


\section{Preliminaries}

Let us first describe the blow-up procedure. We will only sketch this, a full presentation can be found in [19], where this procedure is explained for system (2) (for the scalar case see Gidas-Spruck [24]). Assume that positive solutions of (4) do not have an a priori bound, that is, there exists a sequence $\left(u_{1, n}, u_{2, n}\right)$ of positive solutions of $(4)$ such that at least one of the sequences $u_{1, n}$ and $u_{2, n}$ tends to infinity in the $L^{\infty}$-norm. Let $\beta_{1}, \beta_{2}$ be fixed positive constants to be chosen later. We set

$$
\lambda_{n}=\left\|u_{1, n}\right\|_{L^{\infty}(\Omega)}^{-\beta_{1}},
$$

if $\left\|u_{1, n}\right\|_{L^{\infty}(\Omega)}^{\beta_{2}} \geq\left\|u_{2, n}\right\|_{L^{\infty}(\Omega)}^{\beta_{1}}$ (up to a subsequence), and

$$
\lambda_{n}=\left\|u_{2, n}\right\|_{L^{\infty}(\Omega)}^{-\beta_{2}}
$$

otherwise. We shall suppose - without restricting the generality - that we are in the first of these two situations.

Note that we have $\lambda_{n} \rightarrow 0$ as $n \rightarrow \infty$. Let $x_{n} \in \Omega$ be a point where $u_{1, n}$ assumes its maximum. The functions

$$
v_{i, n}(x)=\lambda_{n}^{\beta_{i}} u_{i, n}\left(\lambda_{n} x+x_{n}\right)
$$

are such that $v_{1, n}(0)=1$ and $0 \leq v_{i, n} \leq 1$ in $\Omega$. One also verifies that the functions $v_{1, n}$ and $v_{2, n}$ satisfy

$$
\left\{\begin{aligned}
-\Delta v_{1, n}= & a(\cdot) \lambda_{n}^{\beta_{1}+2-\beta_{1} \alpha_{11}} v_{1, n}^{\alpha_{11}}+b(\cdot) \lambda_{n}^{\beta_{1}+2-\beta_{2} \alpha_{12}} v_{2, n}^{\alpha_{12}} \\
& +f_{1}(\cdot) \lambda_{n}^{\beta_{1}+2-\beta_{1} \gamma_{11}-\beta_{2} \gamma_{12}} v_{1, n}^{\gamma_{11}} v_{2, n}^{\gamma_{12}}+\lambda_{n}^{\beta_{1}+2} \overline{h_{1, n}} \\
-\Delta v_{2, n}= & c(\cdot) \lambda_{n}^{\beta_{2}+2-\beta_{1} \alpha_{21}} v_{1, n}^{\alpha_{21}}+d(\cdot) \lambda_{n}^{\beta_{2}+2-\beta_{2} \alpha_{22}} v_{2, n}^{\alpha_{22}} \\
& +f_{2}(\cdot) \lambda_{n}^{\beta_{2}+2-\beta_{1} \gamma_{21}-\beta_{2} \gamma_{22}} v_{1, n}^{\gamma_{21}} v_{2, n}^{\gamma_{22}}+\lambda_{n}^{\beta_{2}+2} \overline{h_{2, n}}
\end{aligned}\right.
$$

in the domain $\Omega_{n}=\frac{1}{\lambda_{n}}\left(\Omega-x_{n}\right)$, where the dot stands for $\lambda_{n} x+x_{n}$, and $\widetilde{h_{i, n}}=h_{i}\left(\cdot, \lambda_{n}^{-\beta_{1}} v_{1, n}, \lambda_{n}^{-\beta_{2}} v_{2, n}\right)$. By compactness we can assume that $\left\{x_{n}\right\}$ tends to some point $x_{0} \in \bar{\Omega}$.

The idea is to pass to the limit in (14) and obtain a system which can be proven to have only the trivial solution. This would then contradict the fact that the limit of $v_{1, n}$ has value 1 at the origin.

The next lemma deals with the passage to the limit. 
Lemma 2.1 The sequences $v_{1, n}, v_{2, n}$ converge in $W_{\mathrm{loc}}^{2, p}, 2 \leq p<\infty$ to functions $v_{1}, v_{2} \in C^{2}(G) \cap C^{0}(\bar{G})$, satisfying the limiting system of (14) in $G=\mathbb{R}^{N}$ or $G=\mathbb{R}_{+}^{N}$, provided all the powers of $\lambda_{n}$ in (14) are non-negative. This limiting system is obtained by removing the terms in (14) where the powers of $\lambda_{n}$ are strictly positive, the terms where the coefficient vanishes at $x_{0}$, and the terms containing $h_{i, n}, i=1,2$.

Proof. The argument is standard. The proof of this lemma, except for the last part, can be found in [19]. Note that the passage to the limit in the terms containing products of $v_{1, n}$ and $v_{2, n}$ causes no problem, since $W_{\text {loc }}^{2, p} \cap L^{\infty}$ is an algebra. To prove that the terms in $h_{i, n}$ tend to zero we distinguish two cases : if the sequences $\lambda_{n}^{-\beta_{1}} v_{1, n}$ and $\lambda_{n}^{-\beta_{2}} v_{2, n}$ are both bounded this follows from the local boundedness of $h_{i}$, if one of these sequences is unbounded, it follows from hypothesis (3).

Now we can explain the choice of the couple $\left(\beta_{1}, \beta_{2}\right)$ which we made in the introduction (Cases A, B and C). As stated above, in order to be able to make a passage to the limit in (14) all the powers of $\lambda_{n}$ in this system have to be nonnegative. Hence, geometrically, we have to pick up a point $\left(\beta_{1}, \beta_{2}\right)$ which is to the left of or on $l_{1}$, below or on $l_{2}$, below or on $l_{3}$, and above or on $l_{4}$ (we forget for an instant the mixing terms with coefficients $f_{i}$ ). These are exactly the points which we called admissible - see Figure 1.

Further, it is important to observe that not all admissible points $\left(\beta_{1}, \beta_{2}\right)$ would permit us to prove a priori bounds for positive solutions of (4), as we explain next. Indeed, we have necessarily to make the choice of $\beta_{1}$ and $\beta_{2}$ in such a way that at least two of the powers of $\lambda_{n}$ in (14) are zero. Otherwise, after the passage to the limit in (14) we may end up with an uncoupled system in $\mathbb{R}^{N}$ in which at least one of the equations is the Laplace equation. Hence the corresponding one of the functions $v_{1}$ and $v_{2}$ can be identically equal to a positive constant, and in this way we do not come to a contradiction.

So $\left(\beta_{1}, \beta_{2}\right)$ has to be chosen on the intersection of at least two of the lines $l_{1}, l_{2}, l_{3}, l_{4}$. It can be seen that this is actually possible provided the superlinearity conditions (6) hold. Indeed, the first inequality in (6) implies that the line $l_{1}$ is not empty, and similarly the second one implies that $l_{2}$ is not empty either. Note also that the slope of $l_{3}$ is $\alpha_{12}^{-1}$ and the slope of $l_{4}$ is $\alpha_{21}$; consequently these two lines meet at a point with positive coordinates provided $\alpha_{12} \alpha_{21}>1$.

Observe that the weakly coupled case of [19] corresponds to the situation when the intersection of $l_{1}$ and $l_{2}$ lies strictly below $l_{3}$ and strictly above $l_{4}$, while the strongly coupled case means $l_{3}$ and $l_{4}$ meet in $\Pi$, where $\Pi$ denotes the rectangle enclosed by $l_{1}, l_{2}$, and the axes. We remark that $l_{1}$ and $l_{2}$ can 


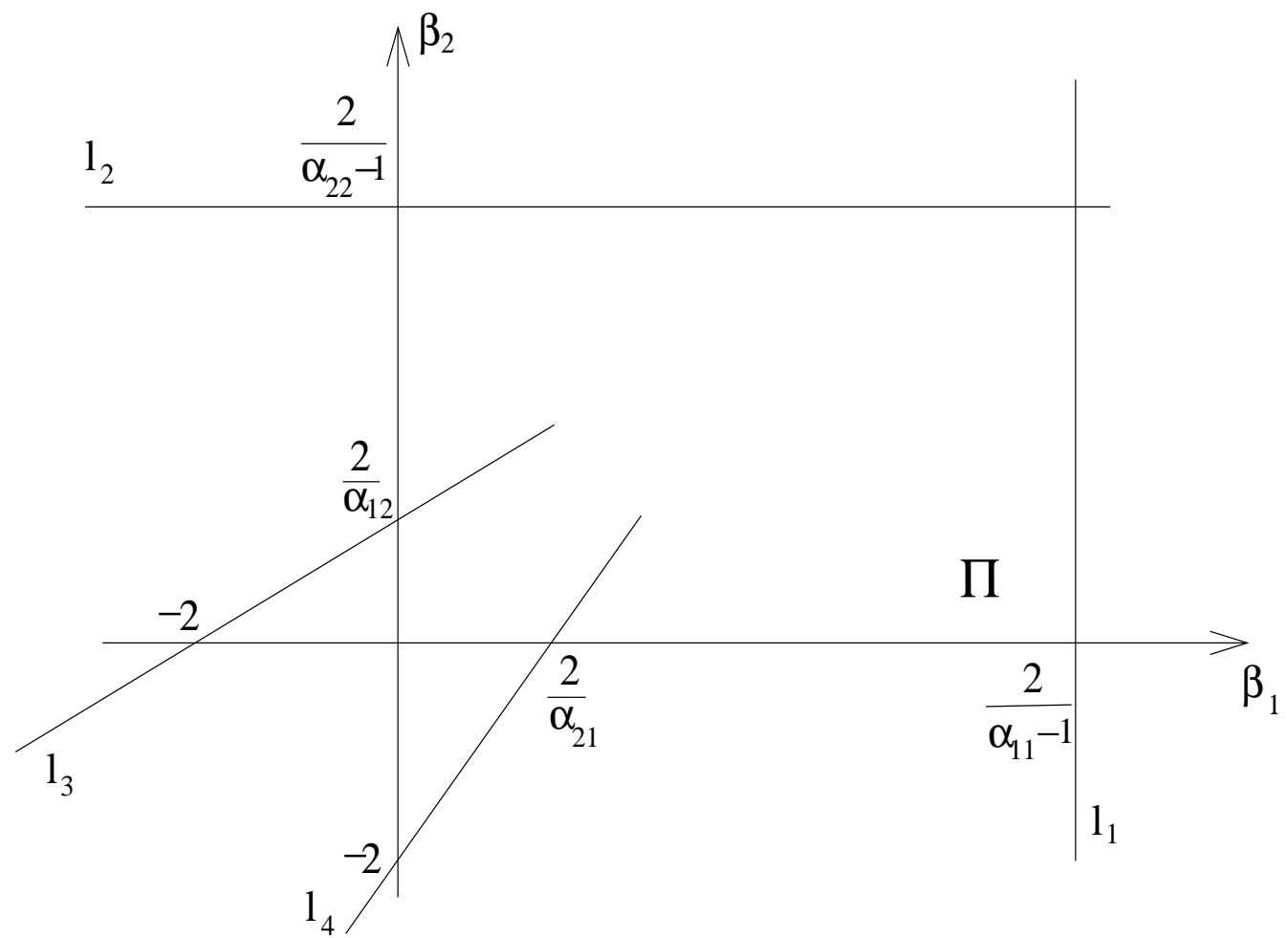

Figure 1: Admissible couples $\left(\beta_{1}, \beta_{2}\right)$ lie to the left of or on $l_{1}$, below or on $l_{2}$, below or on $l_{3}$, and above or on $l_{4}$.

be empty, in case $\alpha_{11} \leq 1$ or $\alpha_{22} \leq 1$; in this case $\Pi$ would be a half strip or the whole positive quadrant of the $\left(\beta_{1}, \beta_{2}\right)$-plane.

The next lemma shows how the terms in (14) with coefficients $f_{i}$ transform after the passage to the limit.

Lemma 2.2 If hypothesis $\left(\gamma_{1}\right)$ holds and $\beta_{1}, \beta_{2}$ are chosen as in the previous section then

$$
\beta_{1}+2-\beta_{1} \gamma_{11}-\beta_{2} \gamma_{12} \geq 0 \quad \text { and } \quad \beta_{2}+2-\beta_{1} \gamma_{21}-\beta_{2} \gamma_{22} \geq 0 .
$$

In Case $A$ the first (resp. the second) inequality in (15) is strict if and only if $l_{3}$ (resp. $l_{4}$ ) does not pass through $l_{1} \cap l_{2}$. In Case $B$ the first (resp. the second) inequality is strict if and only if $l_{1}$ (resp. $l_{2}$ ) does not pass through $l_{3} \cap l_{4}$. In Case $C$ one of the inequalities is always strict and the other is an equality.

Proof. Suppose first that we are in Case A, that is, we have chosen $\left(\beta_{1}, \beta_{2}\right)$ to be the admissible point $l_{1} \cap l_{2}$. This means that

$$
\beta_{1}+2=\alpha_{11} \beta_{1} \geq \alpha_{12} \beta_{2} \quad \text { and } \quad \beta_{2}+2=\alpha_{22} \beta_{2} \geq \alpha_{21} \beta_{1} .
$$


Then we have, by (16) and (5),

$$
\begin{aligned}
\beta_{1}+2-\beta_{1} \gamma_{11}-\beta_{2} \gamma_{12} & =\left(\alpha_{11}-\gamma_{11}\right) \beta_{1}-\gamma_{12} \beta_{2} \\
& =\alpha_{11}\left(1-\frac{\gamma_{11}}{\alpha_{11}}\right) \beta_{1}-\gamma_{12} \beta_{2} \\
& =\gamma_{12}\left(\frac{\alpha_{11}}{\alpha_{12}} \beta_{1}-\beta_{2}\right) \\
& \geq 0
\end{aligned}
$$

and the inequality is strict if and only if $\beta_{1} \alpha_{11}=\beta_{1}+2>\beta_{2} \alpha_{12}$, i.e. $l_{3}$ does not pass through $l_{1} \cap l_{2}$. The second inequality in (15) is proved in a similar way.

Suppose now we are in Case B, that is, we have picked the admissible point $l_{3} \cap l_{4}$ to be our $\left(\beta_{1}, \beta_{2}\right)$. This means

$$
\beta_{1}+2=\alpha_{12} \beta_{2} \geq \alpha_{11} \beta_{1} \quad \text { and } \quad \beta_{2}+2=\alpha_{21} \beta_{1} \geq \alpha_{22} \beta_{2} .
$$

Then, as before,

$$
\begin{aligned}
\beta_{1}+2-\beta_{1} \gamma_{11}-\beta_{2} \gamma_{12} & =-\beta_{1} \gamma_{11}+\left(\alpha_{12}-\gamma_{12}\right) \beta_{2} \\
& =\gamma_{11}\left(-\beta_{1}+\frac{\alpha_{12}}{\alpha_{11}} \beta_{2}\right) \\
& \geq 0,
\end{aligned}
$$

and a similar computation proves the second inequality in (15).

Finally, let us consider Case C. Suppose we have chosen $\left(\beta_{1}, \beta_{2}\right)=l_{1} \cap l_{3}$ (a similar argument can be done when $\left.\left(\beta_{1}, \beta_{2}\right)=l_{2} \cap l_{4}\right)$. Then

$$
\beta_{1}+2=\alpha_{11} \beta_{1}=\alpha_{12} \beta_{2} \quad \text { and } \quad \beta_{2}+2>\max \left\{\alpha_{21} \beta_{1}, \alpha_{22} \beta_{2}\right\},
$$

so $\beta_{1}+2-\beta_{1} \gamma_{11}-\beta_{2} \gamma_{12}=0$ as in Case $\mathrm{A}$. To prove the second inequality in (15) we distinguish two cases. First, if $\alpha_{21} \beta_{1} \geq \alpha_{22} \beta_{2}$ we get

$$
\begin{aligned}
\beta_{2}+2-\beta_{1} \gamma_{21}-\beta_{2} \gamma_{22} & >\left(\alpha_{21}-\gamma_{21}\right) \beta_{1}-\gamma_{22} \beta_{2} \\
& =\gamma_{22}\left(\frac{\alpha_{21}}{\alpha_{22}} \beta_{1}-\beta_{2}\right) \\
& \geq 0,
\end{aligned}
$$

while if $\alpha_{21} \beta_{1} \leq \alpha_{22} \beta_{2}$ we have

$$
\begin{aligned}
\beta_{2}+2-\beta_{1} \gamma_{21}-\beta_{2} \gamma_{22} & >-\beta_{1} \gamma_{21}+\left(\alpha_{22}-\gamma_{22}\right) \beta_{2} \\
& =\gamma_{21}\left(-\beta_{1}+\frac{\alpha_{22}}{\alpha_{21}} \beta_{2}\right) \\
& \geq 0 .
\end{aligned}
$$


This finishes the proof of the Lemma.

We next list the known Liouville type theorems on the equation $-\Delta u=u^{p}$ and on the Lane-Emden system (7). We shall use some of these in the sequel.

Theorem 2.1 The problem

$$
\left\{\begin{array}{r}
-\Delta u=u^{p} \\
u \geq 0
\end{array} \quad \text { in } \quad \mathbb{R}^{N}\right.
$$

has no nontrivial classical solution provided $0<p<\frac{N+2}{N-2}$ (Gidas-Spruck [24], Chen-Li [15]). The same problem has no nontrivial classical supersolution provided $1<p \leq \frac{N}{N-2}$ (Gidas [22], Souto [38], Mitidieri-Pohozaev [32]).

The problem

$$
\left\{\begin{array}{rlll}
-\Delta u & =u^{p} & \text { in } & \mathbb{R}_{+}^{N} \\
u \geq 0 & \text { in } & \mathbb{R}_{+}^{N} \\
u=0 & \text { on } & \partial \mathbb{R}_{+}^{N}
\end{array}\right.
$$

has no nontrivial classical solution provided $1<p<\frac{N+1}{N-3}(1<p<\infty$ if $N=3$ ) - see Gidas-Spruck [24], Dancer [17]. The same problem has no classical supersolution provided $1<p \leq \frac{N+1}{N-1}$ (Bandle-Essen [5], Laptev [29]).

Theorem 2.2 Consider the problem

$$
\left\{\begin{aligned}
-\Delta u & =v^{p} \\
-\Delta v & =u^{q} \quad \text { in } \quad \mathbb{R}^{N} \\
u, v & \geq 0
\end{aligned}\right.
$$

If $p q \leq 1$ (Serrin-Zou [36]) or $0<p, q<\frac{N+2}{N-2}$ (de Figueiredo-Felmer [20]) this problem has no nontrivial classical solutions. If $p q>1$ we set

$$
\alpha=\frac{2(p+1)}{p q-1}, \quad \beta=\frac{2(q+1)}{p q-1} .
$$

Then problem (21) has no nontrivial classical solutions provided either $\max \{\alpha, \beta\} \geq N-2$ (Mitidieri [30], Serrin-Zou [36]), or $p, q>1$ and $\min \{\alpha, \beta\}>\frac{N-2}{2}$ (Busca-Manasevich [10]). The same problem has no nontrivial supersolutions provided $p q>1$ and $\max \{\alpha, \beta\} \geq N-2$ (Mitidieri [31], Laptev [29]). 
The problem

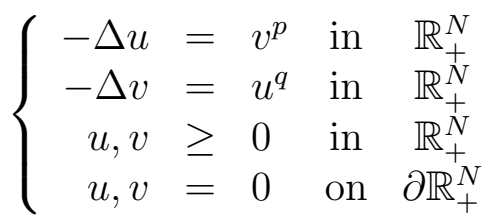

has no nontrivial solutions provided $p, q>1$ and $\max \{\alpha, \beta\} \geq N-3$ (Birindelli-Mitidieri [8]). The same problem does not have nontrivial classical supersolutions provided $p, q>1$ and $\max \{\alpha, \beta\} \geq N-1$ (Laptev [29]).

The problem of non-existence of solutions of Lane-Emden systems in a half-space (system (22)) deserves some discussion. There are two types of results on this problem. On one hand, it is known that a Lane-Emden system in a half-space does not possess positive supersolutions when $\max \{\alpha, \beta\} \geq$ $N-1$. This is an exact result and a particular case of a more general theorem about existence of supersolutions in cones. However, it does not permit to obtain more precise results for solutions. On the other hand, in the framework of a scalar equation Dancer developed a technique, based on the moving planes method, which gives a monotonicity result for bounded solutions of the equation in a half-space. Then one gets as a corollary that the existence of a nontrivial bounded solution in $\mathbb{R}_{+}^{N}$ implies the existence of a non-trivial solution in $\mathbb{R}^{N-1}$. However, the moving planes method requires Lipschitz nonlinearities - that is why a result, where this method is directly employed has to require that all powers involved be greater or equal to one.

The next section contains the proofs of Theorems 1.2 and 1.3, as well as another monotonicity lemma, which will play an important role in the proof of Theorem 1.1.

\section{Monotonicity results for systems}

\subsection{A maximum principle in narrow domains}

In this section we extend to cooperative systems a maximum principle in narrow domains, proved by Cabre [13] in the case of a scalar equation.

We recall the following definition of [13]. For a given domain $\Omega \subset \mathbb{R}^{N}$, the quantity $R(\Omega)$ is defined to be the smallest positive constant $R$ such that

$$
\text { meas }\left(B_{R}(x) \backslash \Omega\right) \geq \frac{1}{2} \operatorname{meas}\left(B_{R}(x)\right), \quad \text { for all } x \in \Omega \text {. }
$$

If no such radius $R$ exists, we define $R(\Omega)=+\infty$. 
It is easy to see that whenever the domain $\Omega$ is contained between two parallel hyperplanes at a distance $d$, we have

$$
R(\Omega) \leq \frac{2^{N} d}{\omega_{N}}
$$

where $\omega_{N}$ is the volume of the unit ball in $\mathbb{R}^{N}$.

In the sequel we shall consider uniformly elliptic second-order operators in the form

$$
L=\sum a_{i j}(x) \partial_{i j}+\sum b_{i}(x) \partial_{i}+c(x),
$$

where $c_{0} I \leq\left(a_{i j}\right) \leq C_{0} I$, sup $\left|b_{i}\right| \leq b$, for some positive constants $c_{0}, C_{0}$, and some $b \geq 0$.

In [13] (see also [14], Theorem 5.3) Cabre proved the following result.

Proposition 3.1 Let $\Omega$ be a domain such that $R(\Omega)<\infty$ and let $L$ be an operator in the form (24), such that $c \leq 0$ in $\Omega$. Suppose $u \in W_{\mathrm{loc}}^{2, N}(\Omega)$ and $f \in L^{\infty}(\Omega)$ satisfy $L u \geq f$ in $\Omega, \limsup _{x \rightarrow \partial \Omega} u(x) \leq 0$, and $\sup _{\Omega} u<\infty$. Then

$$
\sup _{\Omega} u \leq C R(\Omega)^{2}\|f\|_{L^{\infty}(\Omega)},
$$

where $C$ is a constant depending only on $N, c_{0}, C_{0}, b R(\Omega)$.

It is not difficult to deduce from Proposition 3.1 a maximum principle for systems in domains with small $R(\Omega)$.

Theorem 3.1 Let $L_{k}, k=1, \ldots, n$, be uniformly elliptic second-order operators with bounded coefficients and without zero-order term, that is, $L_{k}=$ $\sum a_{i j}^{(k)}(x) \partial_{i j}+\sum b_{i}^{(k)}(x) \partial_{i}, c_{0} I \leq\left(a_{i j}^{(k)}\right) \leq C_{0} I$, sup $\left|b_{i}^{(k)}\right| \leq b$. Let the functions $c_{i j} \in L^{\infty}(\Omega),\left|c_{i j}\right| \leq b$, be such that $c_{i j} \geq 0$ for $i \neq j$. Then there exists a number $\bar{R}$ depending only on $N, c_{0}, C_{0}$ and $b$, such that $R(\Omega) \leq \bar{R}$ implies that each solution $u_{i} \in W_{\mathrm{loc}}^{2, N}(\Omega) \cap C(\bar{\Omega})$ of

$$
\left\{\begin{aligned}
L_{i} u_{i}+\sum_{j=1}^{n} c_{i j} u_{j} & \geq 0 \quad \text { in } \quad \Omega, \quad i=1, \ldots, n \\
u_{i}(x) & \leq 0 \quad \text { on } \partial \Omega, \quad i=1, \ldots, n
\end{aligned}\right.
$$

satisfies $u_{i} \leq 0$ in $\Omega, i=1, \ldots, n$ (in this case we shall say, with obvious notation, that the matrix operator $\vec{L}+\mathcal{C}(x)$ satisfies the maximum principle in $\Omega$ ). 
Proof. For a function $f$ we denote $f^{+}=\max \{f, 0\}, f=f^{+}-f^{-}$. Then $(25)$ implies

$$
L_{i} u_{i}-c_{i i}^{-} u_{i} \geq-c_{i i}^{+} u_{i}^{+}-\sum_{j \neq i} c_{i j} u_{j}^{+}, \quad i=1 \ldots, n .
$$

By applying Proposition 3.1 to these equations we obtain

$$
\sup _{\Omega} u_{i}^{+} \leq C R(\Omega)^{2} \sum_{j=1}^{n} \sup _{\Omega}\left|c_{i j}\right| \sup _{\Omega} u_{j}^{+} \text {. }
$$

Hence, denoting $\vec{v}=\left(\sup _{\Omega} u_{1}^{+}, \ldots, \sup _{\Omega} u_{n}^{+}\right)^{T} \geq 0$ we have

$$
\vec{v} \leq \bar{R}^{2} B \vec{v}
$$

where $B$ is a constant matrix whose entries depend only on $n, N$ and on bounds for the coefficients of the elliptic operators. By choosing $\bar{R}$ such that the matrix $I-\bar{R}^{2} B$ is positive definite and by multiplying scalarly the last inequality by the nonnegative vector $\vec{v}$, we obtain $\vec{v} \equiv 0$.

\subsection{Harnack type estimates for systems}

The moving planes argument in Section 3.3 will require some Harnack estimates which we state in this section. Such results were recently obtained in [12]. The first two theorems below are particular cases of Theorem 3.2 and Propositions 3.1 and 8.1 in [12]. We include them here for the reader's convenience.

In this section $G$ denotes an arbitrary domain in $\mathbb{R}^{N}$ and $Q_{l}(l=1,2)$ are concentric cubes with side $l$, properly included in $G$.

Theorem $3.2([\mathbf{1 2}])$ Assume $f_{1}\left(u_{1}, u_{2}\right), f_{2}\left(u_{1}, u_{2}\right)$ are globally Lipschitz continuous functions, with Lipschitz constant A, which satisfy the cooperativeness assumption (10). Let $\left(u_{1}, u_{2}\right)$ be a nonnegative solution of (9) in $G$. We suppose that the system is fully coupled, in the sense that $f_{1}(0, v)>0$ for all $v>0$, and $f_{2}(u, 0)>0$ for $u>0$. Then for any compact subset $K$ of $G$ there exists a function $\Phi(t)$ (depending on $A, K$ and $G$ ), continuous on $[0, \infty)$, such that $\Phi(0)=0$ and

$$
\sup _{x \in K} \max \left\{u_{1}, u_{2}\right\} \leq \Phi\left(\inf _{x \in K} \min \left\{u_{1}, u_{2}\right\}\right)
$$

In particular, if any of $u_{1}, u_{2}$ vanishes at one point in $G$ then both $u_{1}$ and $u_{2}$ vanish identically in $G$. 
Theorem $3.3([\mathbf{1 2}])$ Assume $f_{1}\left(u_{1}, u_{2}\right), f_{2}\left(u_{1}, u_{2}\right)$ are globally Lipschitz continuous functions, with Lipschitz constant $A$, which satisfy the cooperativeness assumption (10). Let $\left(u_{1}, u_{2}\right)$ be a nonnegative subsolution of (9) in $G$. Then for each $p>0$ there exists a constant $C$ depending only on $p, N$, and A such that

$$
\sup _{x \in Q_{1}} \max \left\{u_{1}, u_{2}\right\} \leq C\left\|\max \left\{u_{1}, u_{2}\right\}\right\|_{L^{p}\left(Q_{2}\right)}
$$

The same result holds if $f_{1}, f_{2}$ depend also on $x$ and the constant $A$ is uniform in $x$.

In the sequel we shall need the following two classical results on scalar equations by Krylov and Safonov.

Theorem 3.4 ([25], Theorem 9.22) Let $L$ be a linear uniformly elliptic operator with bounded coefficients in $G$, in the form (24). Suppose $c_{0}$ is an ellipticity constant for $L$, and $b$ is an upper bound for the $L^{\infty}$-norms of the coefficients of $L$. Let $u \in W_{\mathrm{loc}}^{2, N}(G)$ be a positive function satisfying $L u \leq f$ a.e. in $G$, for some $f \in L_{\mathrm{loc}}^{N}(G)$. Then there exist positive constants $p_{0}$ and $C$ depending on $c_{0}, b$ and $N$ such that

$$
\|u\|_{L^{p_{0}}\left(Q_{2}\right)} \leq C\left(\inf _{x \in Q_{1}} u+\|f\|_{L^{N}\left(Q_{2}\right)}\right) .
$$

Theorem 3.5 (Krylov) Let $L$ be a linear uniformly elliptic operator with bounded coefficients in $G$, in the form (24). Suppose $c_{0}$ is an ellipticity constant for $L$, and $b$ is an upper bound for the $L^{\infty}$-norms of the coefficients of $L$. Let $u \in W_{\mathrm{loc}}^{2, N}(G)$ be a positive function satisfying $L u \leq 0$ a.e. in $G$ and $L u \leq-\rho$ a.e. in a closed subset $\omega \subset Q_{2}$, for some $\rho>0$. Then there exists a constant $m>0$, depending only on $N, c_{0}, b$, and on a positive lower bound on meas $(\omega)>0$, such that

$$
\inf _{Q_{1}} u \geq m \rho
$$

Theorem 3.5 is a consequence of Theorem 12 on p. 129 in [28] - we state it here in the form which was given in [7].

Next we state a partial Harnack inequality for a linear system, which will play a crucial role in the proof of the monotonicity result in Section 3.3.

Theorem 3.6 Suppose $a, b, c, d \in L^{\infty}\left(Q_{2}\right)$ are such that $|a|,|d| \leq A$, and $0 \leq b \leq A, 0 \leq c \leq A$ in $Q_{2}$. Suppose $\left(u_{1}, u_{2}\right)$ is a positive solution of

$$
\left\{\begin{array}{l}
\Delta u_{1}+a(x) u_{1}+b(x) u_{2}=0 \\
\Delta u_{2}+c(x) u_{1}+d(x) u_{2}=0
\end{array}\right.
$$


in $Q_{2}$. Assume in addition that $b(x)$ is bounded below by a positive constant on $Q_{1}$. Then

$$
\sup _{x \in Q_{1}} u_{1} \leq C \inf _{x \in Q_{1}} u_{1}
$$

where the constant $C$ depends on $N, A$, and on upper bound for $\frac{\sup _{Q_{2}} b}{\inf _{Q_{1}} b}$.

Proof. Note that inequality (27) was proved in [12] (Theorem 8.2 in that paper) with the constant $C$ depending on $N, A$, and on constants $\rho>0, \delta>0$ such that $b(x) \geq \rho$ on a set with measure $\delta$. Theorem 3.6 follows from this result applied to the system

$$
\left\{\begin{array}{l}
\Delta u_{1}+a(x) u_{1}+\widetilde{b}(x) \widetilde{u}_{2}=0 \\
\Delta \widetilde{u}_{2}+\widetilde{c}(x) u_{1}+d(x) \widetilde{u}_{2}=0
\end{array}\right.
$$

where $\widetilde{u}_{2}=\left(\inf _{Q_{1}} b\right) u_{2}, \widetilde{c}(x)=\left(\inf _{Q_{1}} b\right) c(x), \widetilde{d}(x)=\left(\inf _{Q_{1}} b\right) d(x)$, and $\widetilde{b}(x)=$ $b(x) /\left(\inf _{Q_{1}} b\right)$, so that $\widetilde{b} \geq 1$ in $Q_{1}$.

We are going to give the argument of the proof of inequality (27), since we shall need it in the sequel. By Theorem 3.3 applied to (28) we have for each $p>0$

$$
\sup _{x \in Q_{1}} \max \left\{u_{1}, \widetilde{u}_{2}\right\} \leq C\left\|\max \left\{u_{1}, \widetilde{u}_{2}\right\}\right\|_{L^{p}\left(Q_{2}\right)} \leq C\left(\left\|u_{1}\right\|_{L^{p}\left(Q_{2}\right)}+\left\|\widetilde{u}_{2}\right\|_{L^{p}\left(Q_{2}\right)}\right) .
$$

In order to estimate the right-hand side of this inequality we apply Theorem 3.4 to the following two scalar inequalities

$$
\left\{\begin{array}{l}
\Delta u_{1}+a(x) u_{1} \leq 0 \\
\Delta \widetilde{u}_{2}+\widetilde{d}(x) \widetilde{u}_{2} \leq 0
\end{array}\right.
$$

(these are a consequence of (28)) and obtain

$$
\sup _{x \in Q_{1}} u_{1} \leq \sup _{x \in Q_{1}} \max \left\{u_{1}, \widetilde{u}_{2}\right\} \leq C\left(\inf _{x \in Q_{1}} u_{1}+\inf _{x \in Q_{1}} \widetilde{u}_{2}\right) .
$$

Finally, we note that the first equation in (28) implies

$$
\Delta u_{1}+a(x) u_{1} \leq 0 \quad \text { in } Q_{2} \quad \text { and } \quad \Delta u_{1}+a(x) u_{1} \leq-\inf _{x \in Q_{1}} \widetilde{u}_{2} \quad \text { in } Q_{1} \text {, }
$$

so Theorem 3.5 gives

$$
\inf _{x \in Q_{1}} u_{1} \geq m \inf _{x \in Q_{1}} \widetilde{u}_{2}
$$

We finish the proof by combining this inequality and (29). 
We finish this section by giving the proof of the strong Harnack inequality for fully coupled systems satisfying hypothesis (11) - Theorem 1.4.

Proof of Theorem 1.4. By (10) and (11) we have

$$
\left\{\begin{array}{l}
\Delta u_{1}+a(x) u_{1}+b(x) u_{2}=0 \\
\Delta u_{2}+c(x) u_{1}+d(x) u_{2}=0
\end{array}\right.
$$

in $Q_{2}$, where the coefficients $a(x)=g_{1}\left(u_{1}(x), u_{2}(x)\right), b(x)=u_{2}^{p-1}(x), c(x)=$ $u_{1}^{q-1}(x), d(x)=g_{2}\left(u_{1}(x), u_{2}(x)\right)$ are bounded continuous functions such that $b(x), c(x)>0$ in $Q_{2}$.

As in the proof of the previous theorem we have

$$
\sup _{x \in Q_{1}} \max \left\{u_{1}, u_{2}\right\} \leq C\left(\inf _{x \in Q_{1}} u_{1}+\inf _{x \in Q_{1}} u_{2}\right) .
$$

By applying Theorem 3.5 to

$$
\Delta u_{1}+a(x) u_{1} \leq 0 \quad \text { in } Q_{2} \quad \text { and } \quad \Delta u_{1}+a(x) u_{1} \leq-\left(\inf _{x \in Q_{1}} u_{2}\right)^{p} \quad \text { in } Q_{1}
$$

we get

$$
\inf _{x \in Q_{1}} u_{1} \geq m\left(\inf _{x \in Q_{1}} u_{2}\right)^{p}
$$

In the same way we obtain

$$
\inf _{x \in Q_{1}} u_{2} \geq m\left(\inf _{x \in Q_{1}} u_{1}\right)^{q}
$$

We obtain the statement of Theorem 1.4 by combining (30) with the last two inequalities.

\subsection{Proof of Theorem $\mathbf{1 . 2}$}

We use the moving planes method of Alexandrov [1], which was subsequently developed in the framework of partial differential equations by Serrin [36], Gidas-Ni-Nirenberg [23], Berestycki-Nirenberg [9].

We shall denote

$$
M=\max \left\{\sup _{\mathbb{R}_{+}^{N}} u_{1}, \sup _{\mathbb{R}_{+}^{N}} u_{2}\right\} .
$$

We can suppose that the functions $f_{1}$ and $f_{2}$ are globally Lipschitz continuous. Indeed, if they are not, we can replace them by $f_{1} \varphi$ and $f_{2} \varphi$, where 
$\varphi$ is a cut-off function such that $\varphi=1$ on the positive cube with side $M$, and $\varphi=0$ outside a cube with side $M+1$, containing properly the previous one.

Hence system (9) satisfies the hypotheses of Theorem 3.2, from which we deduce that either both functions $u_{1}$ and $u_{2}$ vanish identically on $\mathbb{R}_{+}^{N}$ or both $u_{1}$ and $u_{2}$ are strictly positive on $\mathbb{R}_{+}^{N}$. The first case is excluded by hypothesis. So, from now on we shall assume that $u_{1}, u_{2}$ are strictly positive in $\mathbb{R}_{+}^{N}$.

For each $\lambda>0$ we denote

$$
T_{\lambda}=\left\{x \in \mathbb{R}^{N} \mid x_{N}=\lambda\right\}, \quad \Sigma_{\lambda}=\left\{x \in \mathbb{R}^{N} \mid 0<x_{N}<\lambda\right\},
$$

and introduce the functions

$$
v_{i}^{(\lambda)}(x)=u_{i}\left(x^{\prime}, 2 \lambda-x_{N}\right), \quad w_{i}^{(\lambda)}(x)=v_{i}^{(\lambda)}(x)-u_{i}(x), \quad i=1,2,
$$

defined in $\Sigma_{\lambda}$. Since both $\left(u_{1}, u_{2}\right)$ and $\left(v_{1}^{(\lambda)}, v_{2}^{(\lambda)}\right)$ satisfy system (9) we obtain by subtracting the corresponding equations and by Taylor's expansion

$$
\left\{\begin{array}{l}
\Delta w_{1}^{(\lambda)}+c_{11}^{(\lambda)}(x) w_{1}^{(\lambda)}+c_{12}^{(\lambda)}(x) w_{2}^{(\lambda)}=0 \\
\Delta w_{1}^{(\lambda)}+c_{21}^{(\lambda)}(x) w_{1}^{(\lambda)}+c_{22}^{(\lambda)}(x) w_{2}^{(\lambda)}=0
\end{array}\right.
$$

in $\Sigma_{\lambda}$, where $c_{i j}^{\lambda}(x)$ is the partial derivative of $f_{i}$ with respect to $u_{j}$, evaluated at some point between $u_{j}(x)$ and $v_{j}^{(\lambda)}(x)$. Note that $c_{i j}^{(\lambda)}$ are bounded by a Lipschitz constant of $\vec{f}=\left(f_{1}, f_{2}\right)$ on $[0, M]^{2}$, and $c_{12}^{(\lambda)}, c_{21}^{(\lambda)} \geq 0$.

Obviously $\vec{w}^{(\lambda)}=\left(w_{1}^{(\lambda)}, w_{2}^{(\lambda)}\right) \equiv 0$ on $T_{\lambda}$ and $\vec{w}^{(\lambda)}>0$ on $T_{0}$ (recall that $u_{i}=0$ on $T_{0}$ and $u_{i}>0$ on $T_{\lambda}, \lambda>0$ ). By Theorem 3.1, if $\lambda$ is small enough, then $\vec{w}^{(\lambda)} \geq 0$ in $\Sigma_{\lambda}$. Hence

$$
\lambda^{*}=\sup \left\{\lambda \mid \vec{w}^{(\mu)} \geq 0 \text { in } \Sigma_{\mu}, \forall \mu<\lambda\right\}>0 .
$$

We see that for each $0<\lambda \leq \lambda^{*}$ the function $w_{i}^{(\lambda)} \geq 0$ satisfies the inequality $\Delta w_{i}^{(\lambda)}+c_{i i}^{(\lambda)} w_{i}^{(\lambda)} \leq 0$ in $\Sigma_{\lambda}$. Hence Hopf's lemma implies $w_{i}^{(\lambda)}>0$ and

$$
\frac{\partial u_{i}}{\partial x_{N}}=-\frac{1}{2} \frac{\partial w_{i}^{(\lambda)}}{\partial x_{N}}>0 \quad \text { on } T_{\lambda} .
$$

Therefore, the theorem is proved if we show that $\lambda^{*}=+\infty$.

Suppose for contradiction that $\lambda^{*}$ is finite.

By Theorem 3.1 we can fix $\varepsilon_{0}>0$ such that the matrix operator $\vec{\Delta}+C_{\lambda}(x)$ satisfies the maximum principle in the domain $\Sigma_{\lambda^{*}+\varepsilon_{0}} \backslash \Sigma_{\lambda^{*}-\varepsilon_{0}}$ (here $C_{\lambda}(x)$ denotes the matrix of the coefficients in (31)). For instance, we can take

$$
\varepsilon_{0}=\frac{\omega_{N}}{2^{N+1}} \bar{R}
$$

where $\bar{R}$ is the number from Theorem 3.1 (see inequality (23)). 
Lemma 3.1 There exists $\delta_{0} \in\left(0, \varepsilon_{0}\right]$, such that for each $\delta \in\left(0, \delta_{0}\right)$ we have

$$
w_{i}^{\left(\lambda^{*}+\delta\right)} \geq 0 \quad \text { in } \Sigma_{\lambda^{*}-\varepsilon_{0}} \backslash \Sigma_{\varepsilon_{0}}, \quad i=1,2 .
$$

Suppose this lemma is proved. Then we can apply Theorem 3.1 to (31) in $\Sigma_{\lambda^{*}+\delta} \backslash \Sigma_{\lambda^{*}-\varepsilon_{0}}$ and in $\Sigma_{\varepsilon_{0}}$ (these domains are narrow enough) to conclude that $w_{i}^{\left(\lambda^{*}+\delta\right)} \geq 0$ in $\Sigma_{\lambda^{*}+\delta}$ for each $\delta \in\left(0, \delta_{0}\right)$. This contradicts the maximal choice of $\lambda^{*}$ and proves Theorem 1.2.

Proof of Lemma 3.1. We denote $y=\left(x_{1}, \ldots, x_{N-1}\right)$. Suppose for contradiction that there exist sequences $\delta_{m} \rightarrow 0$ and $x^{(m)}=\left(y^{(m)}, x_{N}^{(m)}\right) \in$ $\Sigma_{\lambda^{*}-\varepsilon_{0}} \backslash \Sigma_{\varepsilon_{0}}$ such that

$$
w_{1}^{\left(\lambda^{*}+\delta_{m}\right)}\left(x^{(m)}\right)<0 .
$$

We can suppose that $x_{N}^{(m)} \rightarrow x_{N}^{0} \in\left[\varepsilon_{0}, \lambda^{*}-\varepsilon_{0}\right]$ as $m \rightarrow \infty$.

We define the functions

$$
u_{i}^{(m)}\left(y, x_{N}\right)=u_{i}\left(y+y^{(m)}, x_{N}\right), \quad i=1,2,
$$

and, respectively,

$$
w_{i, \lambda}^{(m)}\left(y, x_{N}\right)=u_{i}^{(m)}\left(y, 2 \lambda-x_{N}\right)-u_{i}^{(m)}\left(y, x_{N}\right), \quad i=1,2 .
$$

Note that system (9) is autonomous, so $\vec{u}^{(m)}$ satisfies the same system. Since $f_{1}\left(u_{1}^{(m)}, u_{2}^{(m)}\right)$ and $f_{2}\left(u_{1}^{(m)}, u_{2}^{(m)}\right)$ are uniformly bounded in $m$ (by a Lipschitz constant of $\vec{f}$ on $[0, M]^{2}$ ), standard elliptic theory, applied to (9), then implies

$$
\left\|\vec{u}^{(m)}\right\|_{W^{2, p}(K)} \leq C M
$$

for each compact set $K$ in the closure of $\mathbb{R}_{+}^{N}$, where $C$ depends on a Lipschitz constant of $\vec{f}$ on $[0, M]^{2}$. It follows, by imbedding theorems and elliptic theory, that $\vec{u}^{(m)}$ converges in $C^{1, \alpha}$ on compact sets to a classical solution $\widetilde{u}=\left(\widetilde{u}_{1}, \widetilde{u}_{2}\right)$ of system $(9)$, satisfying the Dirichlet boundary condition on $\left\{x_{N}=0\right\}$.

It follows from Theorem 3.2 that either both $\widetilde{u}_{1}$ and $\widetilde{u}_{2}$ are strictly positive on $\mathbb{R}_{+}^{N}$ or both these two functions vanish identically on $\mathbb{R}_{+}^{N}$. Suppose that at least one of the functions $\widetilde{u}_{1}, \widetilde{u}_{2}$ does not vanish identically in $\mathbb{R}_{+}^{N}$ so we are in the first of these two situations. By what we have already shown we know that $w_{i, \lambda}^{(m)}\left(y, x_{N}\right)=w_{i}^{\lambda}\left(y+y^{(m)}, x_{N}\right)>0$ in $\Sigma_{\lambda}$ for all $\lambda \leq \lambda^{*}$. Hence the limit functions $\widetilde{w}_{i}^{\lambda}=\lim _{m \rightarrow \infty} w_{i, \lambda}^{(m)}$ are nonnegative in $\Sigma_{\lambda}$ for all $\lambda \leq \lambda^{*}$. However $\widetilde{w}_{i}^{\lambda}$ is to $\widetilde{u}$ what $w_{i}^{\lambda}$ is to $u$, so by repeating the moving planes argument for $\widetilde{u}$ we see that $\widetilde{\lambda}^{*} \geq \lambda^{*}\left(\widetilde{\lambda}^{*}\right.$ is the critical value for $\left.\widetilde{u}\right)$, since we can write 
a system like (31) for $\widetilde{w}_{i}^{\lambda}$. Applying the strong maximum principle to this system we get, as before, that $\widetilde{w}_{i}^{\lambda}>0$ in $\Sigma_{\lambda}$, for $\lambda \leq \lambda^{*}$. On the other hand $\widetilde{w}_{1}^{\lambda^{*}}\left(0, x_{N}^{0}\right)=0$ and $x_{N}^{0} \in\left(0, \lambda^{*}-\varepsilon_{0}\right]$, a contradiction.

The argument is considerably more involved in case $\widetilde{u} \equiv 0$ in $\mathbb{R}_{+}^{N}$. We fix the rectangular domains

$$
\begin{aligned}
& Q_{0}=\left\{x \in \mathbb{R}_{+}^{N} \mid-1<x_{1}<1, \ldots,-1<x_{N-1}<1, \varepsilon_{0}<x_{N}<2 \lambda^{*}+1\right\}, \\
& Q_{1}=\left\{x \in \mathbb{R}_{+}^{N} \mid-2<x_{1}<2, \ldots,-2<x_{N-1}<2, \frac{\varepsilon_{0}}{2}<x_{N}<2 \lambda^{*}+2\right\}, \\
& Q_{2}=\left\{x \in \mathbb{R}_{+}^{N} \mid-3<x_{1}<3, \ldots,-3<x_{N-1}<3, \frac{\varepsilon_{0}}{4}<x_{N}<2 \lambda^{*}+3\right\},
\end{aligned}
$$

and note that all results from the previous section can trivially be applied in $Q_{1}$ and $Q_{2}$ (one simply has to cover $Q_{1}$ and $Q_{2}$ with a finite number of cubes and apply Harnack inequalities in these cubes).

Since $\vec{u}^{(m)}$ converges uniformly to zero in $Q_{2}$ we can suppose that $u_{1}^{(m)} \leq 1$, $u_{2}^{(m)} \leq 1$ in $Q_{2}$. We set

$$
\alpha_{m}=u_{1}^{(m)}\left(0, x_{N}^{(m)}\right), \quad \beta_{m}=u_{2}^{(m)}\left(0, x_{N}^{(m)}\right) .
$$

By Theorem 1.4 we have

$$
\alpha_{m} \leq C \beta_{m}^{\frac{1}{q}} \quad \text { and } \quad \beta_{m} \leq C \alpha_{m}^{\frac{1}{p}},
$$

where $C$ is independent of $m$. We also recall inequality (30)

$$
\sup _{x \in Q_{1}} \max \left\{u_{1}^{(m)}, u_{2}^{(m)}\right\} \leq C\left(\inf _{x \in Q_{1}} u_{1}^{(m)}+\inf _{x \in Q_{1}} u_{2}^{(m)}\right) \text {. }
$$

Next we introduce the functions

$$
z_{1}^{(m)}=\frac{1}{\alpha_{m}} u_{1}^{(m)}, \quad z_{2}^{(m)}=\frac{1}{\beta_{m}} u_{2}^{(m)}, \quad \zeta_{1}^{(m)}=\frac{1}{\beta_{m}} u_{1}^{(m)}, \quad \zeta_{2}^{(m)}=\frac{1}{\alpha_{m}} u_{2}^{(m)} .
$$

Note that $z_{1}^{(m)}\left(0, x_{N}^{(m)}\right)=z_{2}^{(m)}\left(0, x_{N}^{(m)}\right)=1$.

We distinguish two cases : up to a subsequence

$$
\inf _{Q_{1}} u_{1}^{(m)} \leq \inf _{Q_{1}} u_{2}^{(m)} \quad \text { (Case 1) and } \quad \inf _{Q_{1}} u_{2}^{(m)} \leq \inf _{Q_{1}} u_{1}^{(m)} \quad \text { (Case 2). }
$$

Suppose we are in Case 1. Then, as in the previous section, $\left(z_{1}^{(m)}, \zeta_{2}^{(m)}\right)$ satisfies the linear system

$$
\left\{\begin{array}{l}
\Delta z_{1}^{(m)}+a_{m}(x) z_{1}^{(m)}+b_{m}(x) \zeta_{2}^{(m)}=0 \\
\Delta \zeta_{2}^{(m)}+c_{m}(x) z_{1}^{(m)}+d_{m}(x) \zeta_{2}^{(m)}=0
\end{array}\right.
$$


in $Q_{2}$, where $a_{m}(x)=g_{1}\left(u_{1}^{(m)}(x), u_{2}^{(m)}(x)\right), b_{m}(x)=\left(u_{2}^{(m)}(x)\right)^{p-1}>0$, $c_{m}(x)=\left(u_{1}^{(m)}(x)\right)^{q-1}>0, d_{m}(x)=g_{2}\left(u_{1}^{(m)}(x), u_{2}^{(m)}(x)\right)$ are uniformly bounded in $m$.

By combining inequality (34) with the hypothesis of Case 1 we obtain

$$
\sup _{Q_{1}} u_{2}^{(m)} \leq C \inf _{Q_{1}} u_{2}^{(m)}
$$

which implies that the quantity

$$
\frac{\sup _{x \in Q_{1}} b_{m}(x)}{\inf _{x \in Q_{1}} b_{m}(x)}
$$

is bounded by a constant independent of $m$. Hence we can apply Theorem 3.6 to (35), and infer that

$$
\sup _{Q_{0}} z_{1}^{(m)} \leq C_{1} \inf _{Q_{0}} z_{1}^{(m)} \leq C_{1}
$$

where $C_{1}$ does not depend on $m$.

Next, recall that $w_{1}^{\left(\lambda^{*}\right)} \geq 0$ in $\Sigma_{\lambda^{*}}$, which implies

$$
z_{1}^{(m)}\left(y, x_{N}\right) \leq z_{1}^{(m)}\left(y, 2 \lambda^{*}-x_{N}\right) \leq C_{1}
$$

for every $\left(y, x_{N}\right)$ in the closure of $\Sigma_{\lambda^{*}}$. Hence

$$
\left\|z_{1}^{(m)}\right\|_{L^{\infty}(Q)} \leq C_{1},
$$

where

$$
Q=\left\{x \in \mathbb{R}_{+}^{N} \mid-1<x_{1}<1, \ldots,-1<x_{N-1}<1,0<x_{N}<2 \lambda^{*}+1\right\} .
$$

In Case 2 we write a linear system for $\left(\zeta_{1}^{(m)}, z_{2}^{(m)}\right)$ and use an analogous reasoning to infer that

$$
\left\|z_{2}^{(m)}\right\|_{L^{\infty}(Q)} \leq C_{2}
$$

where $C_{2}$ does not depend on $m$.

Next we prove that actually both $z_{1}^{(m)}$ and $z_{2}^{(m)}$ are bounded in $L^{\infty}(Q)$. To this end we remark that $\left(z_{1}^{(m)}, z_{2}^{(m)}\right)$ satisfies the system

$$
\left\{\begin{aligned}
\Delta z_{1}^{(m)}+g_{1}\left(u_{1}^{(m)}, u_{2}^{(m)}\right) z_{1}^{(m)}+\frac{\beta_{m}^{p}}{\alpha_{m}}\left(z_{2}^{(m)}\right)^{p}=0 \text { in } \mathbb{R}_{+}^{N} \\
\Delta z_{2}^{(m)}+\frac{\alpha_{m}^{q}}{\beta_{m}}\left(z_{1}^{(m)}\right)^{q}+g_{1}\left(u_{1}^{(m)}, u_{2}^{(m)}\right) z_{2}^{(m)}=0 \text { in } \mathbb{R}_{+}^{N} \\
z_{1}^{(m)}=z_{2}^{(m)}=0 \text { on } \partial \mathbb{R}_{+}^{N}
\end{aligned}\right.
$$


by (11). We recall that we have already proved that the sequences $\left\{\beta_{m}^{p} \alpha_{m}^{-1}\right\}$ and $\left\{\alpha_{m}^{q} \beta_{m}^{-1}\right\}$ are bounded (see (33)). It follows from the above argument that one of the sequences $\left\{z_{1}^{(m)}\right\},\left\{z_{2}^{(m)}\right\}$ (say the first) is bounded in $L^{\infty}(Q)$. Then standard elliptic estimates applied to the second equation in (36), regarded as a scalar equation in $z_{2}^{(m)}$ with bounded coefficients and bounded right-hand side, imply that $\left\{z_{2}^{(m)}\right\}$ is also bounded in $L^{\infty}(Q)$ (if necessary, we restrict a little the upper and the lateral boundaries of $Q)$.

Now, since both $\left\{z_{1}^{(m)}\right\},\left\{z_{2}^{(m)}\right\}$ are bounded on Q, elliptic theory and (36) imply that (up to a subsequence) these two sequences converge uniformly to nonnegative functions $z_{1}, z_{2} \in W_{\text {loc }}^{2, p}(Q) \cap C(\bar{Q})$, which satisfy the system

$$
\left\{\begin{aligned}
\Delta z_{1}+g_{1}(0,0) z_{1}+\beta_{0}\left(z_{2}\right)^{p} & =0 \text { in } Q \\
\Delta z_{2}+\alpha_{0}\left(z_{1}\right)^{q}+g_{1}(0,0) z_{2} & =0 \text { in } Q \\
z_{1}=z_{2} & =0 \text { on }\left\{x_{N}=0\right\} \cap \partial Q,
\end{aligned}\right.
$$

for some constants $\alpha_{0} \geq 0, \beta_{0} \geq 0$. Since $z_{1} \geq 0$ and $\Delta z_{1}+g_{1}(0,0) z_{1} \leq 0$ in $Q$, the strong maximum principle implies that either $z_{1}$ vanishes identically in $Q$ or $z_{1}>0$ in $Q$. The first possibility is excluded by $z_{1}\left(0, x_{N}^{0}\right)=1$.

Introduce the comparison functions

$$
\omega_{i}^{(\lambda)}\left(y, x_{N}\right)=z_{i}\left(y, 2 \lambda-x_{N}\right)-z_{i}\left(y, x_{N}\right), \quad, i=1,2,
$$

defined in $\Sigma_{\lambda} \cap \bar{Q}$, for all $\lambda \leq \lambda^{*}+1 / 2$. We have, by continuity,

$$
\omega_{i}^{\left(\lambda^{\star}\right)} \geq 0, i=1,2, \quad \text { and } \quad \omega_{1}^{\left(\lambda^{\star}\right)}\left(0, x_{N}^{0}\right)=0
$$

(recall (32)). Since $\Delta \omega_{1}^{\left(\lambda^{\star}\right)}+g_{1}(0,0) \omega_{1}^{\left(\lambda^{\star}\right)} \leq 0$ the strong maximum principle implies $\omega_{1}^{\left(\lambda^{\star}\right)} \equiv 0$ in $\Sigma_{\lambda^{*}} \cap \bar{Q}$. This contradicts the fact that $z_{1}=0$ on $\left\{x_{N}=0\right\}$ and $z_{1}>0$ on $\left\{x_{N}=2 \lambda^{*}\right\}$.

The proof of Theorem 1.2 is finished.

\subsection{Proof of Theorem 1.3}

Set

$$
x^{\prime} \in \mathbb{R}^{N-1}, \quad u_{i}^{(t)}\left(x^{\prime}\right)=u_{i}\left(x^{\prime}, t\right), \quad i=1,2 .
$$

Since the sequence $\left\{u_{i}^{(t)}\right\}_{t}$ is uniformly bounded and pointwise increasing in $t$ (by Theorem 1.2) the Lebesgue monotone convergence theorem implies that $u_{i}^{(t)}$ converges as $t \rightarrow \infty$ in $L_{\text {loc }}^{p}\left(\mathbb{R}^{N-1}\right), p<\infty$, to a bounded function $\widetilde{u}_{i}$. Then the hypotheses we made on $f_{i}$ imply

$$
f_{i}\left(u_{1}^{(t)}, u_{2}^{(t)}\right) \longrightarrow f_{i}\left(\widetilde{u_{1}}, \widetilde{u_{2}}\right) \quad \text { as } t \rightarrow \infty,
$$


in $L_{\text {loc }}^{p}\left(\mathbb{R}^{N-1}\right), p<\infty, i=1,2$. We shall prove that $\left(\widetilde{u_{1}}, \widetilde{u_{2}}\right)$ is a weak solution to the problem in $\mathbb{R}^{N-1}$ (then standard elliptic regularity theory implies that it is a classical solution). Let $\phi\left(x^{\prime}\right) \in C_{\mathrm{c}}^{\infty}\left(\mathbb{R}^{N-1}\right)$ be an arbitrary function and let

$$
\psi(t)=\left\{\begin{array}{ccc}
0 & \text { if } & t \leq 0, t \geq 2 \\
t & \text { if } & 0 \leq t \leq 1 \\
2-t & \text { if } & 1 \leq t \leq 2
\end{array}\right.
$$

The function $\psi(t)$ is chosen so that $\psi \in C_{\mathrm{c}}(\mathbb{R}), \operatorname{supp} \psi=[0,2]$,

$$
\int_{0}^{1} \psi(t) d t=\int_{1}^{2} \psi(t) d t=\frac{1}{2} \quad \text { and } \quad \psi^{\prime \prime}(t)=\delta(0)-2 \delta(1)+\delta(2),
$$

where $\delta(t)$ is the Dirac mass at $t$. We set $\psi_{m}(t)=\psi(t-m)$.

We multiply each equation in (9) by $\phi\left(x^{\prime}\right) \psi_{m}\left(x_{N}\right)$ and integrate over $\mathbb{R}_{+}^{N}$. Integration by parts and Fubini's theorem then yield

$$
\begin{aligned}
\int_{\mathbb{R}_{+}^{N}} u_{i}\left(\Delta_{x^{\prime}} \phi\left(x^{\prime}\right) \psi_{m}\left(x_{N}\right)\right. & \left.+\phi\left(x^{\prime}\right) \psi_{m}^{\prime \prime}\left(x_{N}\right)\right) d x_{N} d x^{\prime} \\
& =\int_{\mathbb{R}_{+}^{N}} f_{i}\left(u_{1}, u_{2}\right) \phi\left(x^{\prime}\right) \psi_{m}\left(x_{N}\right) d x_{N} d x^{\prime}
\end{aligned}
$$

or

$$
\begin{aligned}
& \int_{\mathbb{R}^{N-1}}\left(\int_{0}^{2} u_{i}\left(x^{\prime}, x_{N}+m\right) \psi\left(x_{N}\right) d x_{N}\right) \Delta_{x^{\prime}} \phi\left(x^{\prime}\right) d x^{\prime} \\
& +\int_{\mathbb{R}^{N-1}}\left(u_{i}^{(m)}\left(x^{\prime}\right)-2 u_{i}^{(m+1)}\left(x^{\prime}\right)+u_{i}^{(m+2)}\left(x^{\prime}\right)\right) \phi\left(x^{\prime}\right) d x^{\prime} \\
& =\int_{\mathbb{R}^{N-1}}\left(\int_{0}^{2} f_{i}\left(u_{1}\left(x^{\prime}, x_{N}+m\right), u_{2}\left(x^{\prime}, x_{N}+m\right)\right) \psi\left(x_{N}\right) d x_{N}\right) \phi\left(x^{\prime}\right) d x^{\prime} .
\end{aligned}
$$

Since $u_{i}^{(m)}$ tends to $\widetilde{u_{i}}$ in any Lebesgue space, the following lemma finishes the proof of Theorem 1.3. Note that the second integral in the left-hand side of the last equality vanishes at the limit.

Lemma 3.2 Under the hypotheses we made on $f_{1}, f_{2}$

$$
\int_{0}^{2} f_{i}\left(u_{1}\left(x^{\prime}, x_{N}+m\right), u_{2}\left(x^{\prime}, x_{N}+m\right)\right) \psi\left(x_{N}\right) d x_{N}
$$

tends to $f_{i}\left(\widetilde{u_{1}}\left(x^{\prime}\right), \widetilde{u_{2}}\left(x^{\prime}\right)\right)$ in $L_{\text {loc }}^{1}\left(\mathbb{R}^{N-1}\right)$ as $m \rightarrow \infty$. 
Proof. We split the integral (38) in two and use the fact that $\psi$ is continuous and monotonous in $[0,1]$ and in $[1,2]$. By standard properties of the Riemann integral (38) is equal to

$$
\frac{f\left(u_{1}\left(x^{\prime}, \xi_{1}+m\right), u_{2}\left(x^{\prime}, \xi_{1}+m\right)\right)+f\left(u_{1}\left(x^{\prime}, \xi_{2}+m\right), u_{2}\left(x^{\prime}, \xi_{2}+m\right)\right)}{2}
$$

where $\xi_{1} \in(0,1), \xi_{2} \in(1,2)$. Obviously $u_{i}\left(x^{\prime}, \xi_{j}+m\right)$ tends to $\widetilde{u_{i}}\left(x^{\prime}\right)$ in $L_{\text {loc }}^{p}\left(\mathbb{R}^{N-1}\right), p<\infty$, so the lemma follows.

\subsection{A monotonicity lemma}

In the proof of Theorem 1.1 we shall need the following monotonicity result, which is an extension of a recent result of Busca and Manasevich [10].

Lemma 3.3 Let $f_{i} \in C^{1}\left(\left(\mathbb{R}_{+}\right)^{2}\right), f_{i}(0,0)=0, \nabla f_{i}(0,0)=0, i=1,2$ satisfy (10). Suppose that $f_{1}(0, v)>0$ for all $v>0$, and $f_{2}(u, 0)>0$ for $u>0$. Let $u_{i}(t, \theta) \geq 0, i=1,2$ be $C^{2}$-functions defined on $\mathbb{R} \times S^{N-1}$ and satisfying

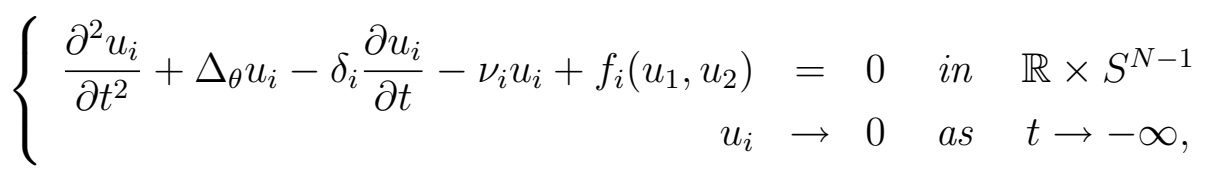

where $\delta_{i} \geq 0, \max \left\{\delta_{1}, \delta_{2}\right\}>0, \nu_{i}>0, i=1,2$ are constants. Suppose also that there exists $t_{0} \in \mathbb{R}$ such that $\frac{\partial u_{i}}{\partial t}>0$ in $\left(-\infty, t_{0}\right) \times S^{N-1}$, for $i=1,2$. Then $\frac{\partial u_{i}}{\partial t}>0$ in $\mathbb{R} \times S^{N-1}$, for $i=1,2$.

In [10] the authors studied the case $n=2, f_{1}\left(u_{1}, u_{2}\right)=u_{2}^{p}, f_{2}\left(u_{1}, u_{2}\right)=u_{1}^{q}$. Note that the result in [10] was stated without the hypothesis $p, q>1$, but this hypothesis is actually used in their proof.

The proof of Lemma 3.3 is based on the moving planes method and uses both ideas from the proof in [10] and from the proof of the symmetry result for systems in $\mathbb{R}^{N}$ obtained in [11].

We set

$$
h_{i}\left(u_{1}, u_{2}\right)=-\nu_{i} u_{i}+f_{i}\left(u_{1}, u_{2}\right)
$$

and remark that all we shall need is the fact that the matrix of the partial derivatives of $h_{1}$ and $h_{2}$ is negative definite at $(0,0)$. We use this fact as in the proof of Theorem 2 from [11] (see hypotheses (i)-(iii) in Section 2.1 of [11]).

For each $\lambda>0$ we denote

$$
T_{\lambda}=\left\{(t, \theta) \in \mathbb{R} \times S^{N-1} \mid t=\lambda\right\}, \quad \Sigma_{\lambda}=\left\{(t, \theta) \in \mathbb{R} \times S^{N-1} \mid t<\lambda\right\},
$$


and introduce the functions

$$
w_{i}^{(\lambda)}(t, \theta)=u_{i}(2 \lambda-t, \theta)-u_{i}(t, \theta), \quad i=1, \ldots, n,
$$

defined in $\Sigma_{\lambda}$. By Taylor's expansion $\vec{w}_{\lambda}$ satisfies the system

$$
\frac{\partial^{2} \vec{w}^{(\lambda)}}{\partial t^{2}}+\Delta_{\theta} \vec{w}^{(\lambda)}-D \frac{\partial \vec{w}^{(\lambda)}}{\partial t}+C_{\lambda}(x) \vec{w}^{(\lambda)}=-2 D \frac{\partial \vec{u}}{\partial t} \quad \text { in } \quad \Sigma_{\lambda},
$$

where

$$
D=\operatorname{diag}\left(\delta_{1}, \delta_{2}\right), \quad C_{\lambda}(x)=\left(c_{i j}(x)\right)_{i, j=1}^{2}, \quad c_{i j}(x)=\frac{\partial h_{i}}{\partial u_{j}}\left(\xi_{1}, \xi_{2}\right),
$$

and $\xi_{i j}=\xi_{i j}(t, \theta, \lambda)$,

$$
\xi_{i j} \in\left[\min \left(u_{j}(t, \theta), u_{j}(2 \lambda-t, \theta)\right), \max \left(u_{j}(t, \theta), u_{j}(2 \lambda-t, \theta)\right)\right] .
$$

System (40) is treated in basically the same way as inequality (3)-(4) from [11] - note that the right hand side of (40) is nonpositive in $\Sigma_{\lambda}$ for any $\lambda \leq t_{0}$, by hypothesis. We shall only sketch the argument, since most of the details can be seen in [11] (note only that here the moving planes go "to the right", contrary to the choice made in [11]). First, there exists $\lambda^{*}<t_{0}$ such that $\vec{w}^{(\lambda)} \geq 0$ in $\Sigma_{\lambda}$, for any $\lambda \leq \lambda^{*}$. The proof of this fact goes like the proof of Step 1 in the proof of Theorem 2 in [11]. Second, we define

$$
\lambda_{0}=\sup \left\{\lambda \in \mathbb{R}: \vec{w}^{(\mu)} \geq 0 \quad \text { and } \quad \frac{\partial \vec{w}^{(\mu)}}{\partial t}>0 \text { in } \Sigma_{\mu}, \quad \text { for all } \mu<\lambda\right\} \text {. }
$$

If $\lambda_{0}=+\infty$ we are done (note that $\frac{\partial \vec{w}^{(\lambda)}}{\partial t}=2 \frac{\partial \vec{u}}{\partial t}$ on $T_{\lambda}$ ). If $\lambda_{0}$ is finite we reason like in Step 2 in the proof of Theorem 2 in [11], to infer that either $w_{1}^{\left(\lambda_{0}\right)}$ or $w_{2}^{\left(\lambda_{0}\right)}$ vanishes in $\Sigma_{\lambda_{0}}$. Since the system is fully coupled this implies that $\vec{w}^{\left(\lambda_{0}\right)} \equiv 0$ in $\Sigma_{\lambda_{0}}$, or, equivalently, $\frac{\partial \vec{u}}{\partial t}$ is odd with respect to $T_{\lambda_{0}}$. On the other hand, by (40) at least one of the derivatives with respect to $t$ of $u_{1}$ and $u_{2}$ (the one which corresponds to a strictly positive $\delta_{i}$ ) is even with respect to $T_{\lambda_{0}}$. Hence at least one of the functions $u_{i}$ is equal to a constant, which contradicts the hypothesis that $u_{i}$ is strictly increasing in $t$ for $t \in\left(-\infty, t_{0}\right)$.

In the next section we prove our main result. 


\section{$4 \quad$ Proof of Theorem 1.1}

We divide the proof in three parts, according to the case we consider (A, B or $\mathrm{C}$ ). We recall that the values of the parameters $\alpha_{i j}$ determine which case we are in.

In the sequel $G$ will denote either $\mathbb{R}^{N}$ or $\mathbb{R}_{+}^{N}$.

Proof in Case A. In this case we choose $\left(\beta_{1}, \beta_{2}\right)=l_{1} \cap l_{2}$, that is

$$
\beta_{1}=\frac{2}{\alpha_{11}-1}, \quad \beta_{2}=\frac{2}{\alpha_{22}-1} .
$$

1. First, if none of the lines $l_{3}$ and $l_{4}$ passes through $l_{1} \cap l_{2}$ we are precisely in the weakly coupled case considered in [19]. By using Lemmas 2.1 and 2.2, and by letting $n \rightarrow \infty$ in (14) we obtain (after scaling) the uncoupled system

$$
\left\{\begin{array}{l}
-\Delta v_{1}=v_{1}^{\alpha_{11}} \\
-\Delta v_{2}=v_{2}^{\alpha_{22}}
\end{array}\right.
$$

in $G$, which has only the trivial solution because

$$
\max \left\{\alpha_{11}, \alpha_{22}\right\}<\frac{N+2}{N-2},
$$

which is a consequence of (8) and (41).

2. Next, suppose exactly one of $l_{3}$ and $l_{4}$ (say $l_{3}$ ) passes through $l_{1} \cap l_{2}$. Then again by Lemmas 2.1 and 2.2, after letting $n \rightarrow \infty$ in (14) we obtain the system

$$
\left\{\begin{aligned}
-\Delta v_{1} & =a_{0} v_{1}^{\alpha_{11}}+b_{0} v_{2}^{\alpha_{12}}+c_{1} v_{1}^{\gamma_{11}} v_{2}^{\gamma_{12}} \\
-\Delta v_{2} & =d_{0} v_{2}^{\alpha_{22}} \quad \text { in } G \\
u, v & \geq 0
\end{aligned}\right.
$$

where $a_{0}, d_{0}, b_{0}, c_{1}$ are constants such that $a_{0}, d_{0}>0, b_{0}, c_{1} \geq 0$. Since (43)

holds this system has no nontrivial solution. Indeed, the second equation in (44) implies $v_{2} \equiv 0$. Then the first equation becomes a scalar equation, which again has no nontrivial solutions under (43).

3. Finally, suppose all four lines $l_{1}, l_{2}, l_{3}, l_{4}$ meet at one point (so we are simultaneously in Cases A and B). Letting $n \rightarrow \infty$ in (14), we come to the system

$$
\left\{\begin{aligned}
-\Delta v_{1} & =a_{0} v_{1}^{\alpha_{11}}+b_{0} v_{2}^{\alpha_{12}}+c_{1} v_{1}^{\gamma_{11}} v_{2}^{\gamma_{12}} \\
-\Delta v_{2} & =c_{0} v_{1}^{\alpha_{21}}+d_{0} v_{2}^{\alpha_{22}}+c_{2} v_{1}^{\gamma_{21}} v_{2}^{\gamma_{22}} \quad \text { in } G \\
u, v & \geq 0
\end{aligned}\right.
$$


where $a_{0}, b_{0}, c_{0}, d_{0}>0, c_{1}, c_{2} \geq 0$. Note that now

$$
\alpha_{12}=\frac{\alpha_{11}\left(\alpha_{22}-1\right)}{\alpha_{11}-1}, \quad \alpha_{21}=\frac{\alpha_{22}\left(\alpha_{11}-1\right)}{\alpha_{22}-1} .
$$

Assume first $G=\mathbb{R}_{+}^{N}$. Then (45) satisfies the hypotheses of Theorem 1.2 and Theorem 1.3. Hence, by Theorem 1.3, if $\left(v_{1}, v_{2}\right) \not \equiv(0,0)$ then there exists a nontrivial solution of (45) in $\mathbb{R}^{N-1}$. So, if we manage to prove that (45) has only the trivial solution in $\mathbb{R}^{N}$ under (8) (note that a particular case of this result would be Theorem 1.5), then it has no nontrivial solutions in $\mathbb{R}_{+}^{N}$ under $\min \left\{\beta_{1}, \beta_{2}\right\}>\frac{N-3}{2}$ which is consequence of (8).

From now on we suppose $G=\mathbb{R}^{N}$ and distinguish two cases, $\max \left\{\beta_{1}, \beta_{2}\right\} \geq N-2$ (Case 1) and $\max \left\{\beta_{1}, \beta_{2}\right\}<N-2$ (Case 2).

In Case 1 ( say $\beta_{1} \geq N-2$ ) we have $\alpha_{11} \leq \frac{N}{N-2}$. But the first equality in (45) implies $-\Delta v_{1} \geq a_{0} v_{1}^{\alpha_{11}}$ in $\mathbb{R}^{N}$, so $v_{1} \equiv 0$ in $\mathbb{R}^{N}$, by the results about non-existence of supersolutions (Theorem 2.1). Then the second equation in (45) becomes $-\Delta v_{2}=d_{0} v_{2}^{\alpha_{22}}$ in $\mathbb{R}^{N}$. So $v_{2} \equiv 0$ in $\mathbb{R}^{N}$, because $\alpha_{22}<\frac{N+2}{N-2}$, which is a consequence of (8).

In Case 2 we write system (45) in polar coordinates $(r, \theta) \in \mathbb{R} \times S^{N-1}$ and make the change of variables, as in [10],

$$
r=|x|, \quad t=\ln |x| \in \mathbb{R}, \quad \theta=\frac{x}{|x|} \in S^{N-1},
$$

and set

$$
w_{i}(t, \theta)=e^{\beta_{i} t} v_{i}\left(e^{t}, \theta\right) .
$$

Then system (45) transforms into

$$
\left\{\begin{aligned}
-L_{1} w_{1}= & a_{0} e^{\left(\beta_{1}+2-\alpha_{11} \beta_{1}\right) t} w_{1}^{\alpha_{11}}+b_{0} e^{\left(\beta_{1}+2-\alpha_{12} \beta_{2}\right) t} w_{2}^{\alpha_{12}} \\
& +c_{1} e^{\left(\beta_{1}+2-\gamma_{11} \beta_{1}-\gamma_{12} \beta_{2}\right) t} w_{1}^{\gamma_{11}} w_{2}^{\gamma_{12}} \\
-L_{2} w_{2}= & c_{0} e^{\left(\beta_{2}+2-\alpha_{21} \beta_{1}\right) t} w_{1}^{\alpha_{21}}+d_{0} e^{\left(\beta_{2}+2-\alpha_{22} \beta_{2}\right) t} w_{2}^{\alpha_{22}} \\
& +c_{2} e^{\left(\beta_{2}+2-\gamma_{21} \beta_{1}-\gamma_{22} \beta_{2}\right) t} w_{1}^{\gamma_{21}} w_{2}^{\gamma_{22}}
\end{aligned}\right.
$$

in $\mathbb{R} \times S^{N-1}$, where

$$
L_{i}=\frac{\partial^{2}}{\partial t^{2}}+\Delta_{\theta}-\delta_{i} \frac{\partial}{\partial t}-\nu_{i}, \quad i=1,2,
$$

and

$$
\delta_{i}=2 \beta_{i}-(N-2), \quad \nu_{i}=\beta_{i}\left(N-2-\beta_{i}\right), \quad i=1,2 .
$$


It is remarkable that after this change of variables one obtains the same powers in the exponential functions in (46) as the powers of the parameter $\lambda_{n}$ after the "blow-up" change of variables. So, in the case we consider all these powers are zero, and system (46) is autonomous.

Observe that condition (8) implies that $\delta_{i}>0$, and that the condition of Case 2 gives $\nu_{i}>0$. Further, by by applying Theorem 3.2 to system (45) we see that we can suppose that $v_{1}$ and $v_{2}$ are strictly positive in $\mathbb{R}^{N}$. This easily implies that the derivatives of $w_{1}$ and $w_{2}$ with respect to $t$ are positive for large negative $t$. So we can now apply Lemma 3.3 to (46), and infer $\frac{\partial w_{i}}{\partial t}>0$ in $\mathbb{R} \times S^{N-1}$, or

$$
\beta_{i} v_{i}+r \frac{\partial v_{i}}{\partial r}>0, \quad i=1,2
$$

Then, using a reasoning from [10], we remark that all the above argument can be carried out for any translation of $\left(v_{1}, v_{2}\right)$ (since (45) is autonomous), so (47) implies

$$
\beta_{i} v_{i}(x)+\nabla v_{i}(x)\left(x-x_{0}\right) \geq 0,
$$

for all $x, x_{0} \in \mathbb{R}^{N}$. This easily implies $\nabla v_{i}(x) \equiv 0$ (write $x_{0}=x-\tau e, \tau>0$, $e \in S^{N-1}$, divide by $\tau$, let $\tau \rightarrow \infty$ and observe that the resulting inequality holds for any $e \in S^{N-1}$ ), which contradicts (45).

Proof in Case B. We recall that in this case we choose $\left(\beta_{1}, \beta_{2}\right)=l_{3} \cap l_{4}$, that is,

$$
\beta_{1}=\frac{2\left(1+\alpha_{12}\right)}{\alpha_{12} \alpha_{21}-1}, \quad \beta_{2}=\frac{2\left(1+\alpha_{21}\right)}{\alpha_{12} \alpha_{21}-1} .
$$

1. First, suppose none of $l_{1}$ and $l_{2}$ passes through $l_{3} \cap l_{4}$; this is precisely the strongly coupled case from [19]. Then by using Lemmas 2.1 and 2.2, after the passage to the limit in (14) we obtain (after scaling) the system

$$
\left\{\begin{aligned}
-\Delta v_{1} & =v_{2}^{\alpha_{12}} \\
-\Delta v_{2} & =v_{1}^{\alpha_{21}} \\
u, v & \geq 0
\end{aligned} \quad \text { in } \quad G\right.
$$

Observe that it is known that under (8) system (48) in $\mathbb{R}^{N}$ has only the trivial solution, see Theorem 2.2. However, as in the beginning of point 3 of the proof of Case A above we see that we can restrict the analysis to $G=\mathbb{R}^{N}$. Indeed, system (48) satisfies the hypotheses of Theorem 1.2, since by assumption $b_{0}, c_{0}>0, \alpha_{12}, \alpha_{21}>1$. Let us stress again that this argument requires Lipschitz nonlinearities.

2. Second, if one of $l_{1}$ or $l_{2}$ (say $l_{1}$ ) passes through $l_{3} \cap l_{4}$, by letting $n \rightarrow \infty$ in (14) we obtain, again with the help of Lemmas 2.1 and 2.2, the 
following system

$$
\left\{\begin{aligned}
-\Delta v_{1} & =a_{0} v_{1}^{\alpha_{11}}+b_{0} v_{2}^{\alpha_{12}}+c_{1} v_{1}^{\gamma_{11}} v_{2}^{\gamma_{12}} \\
-\Delta v_{2} & =c_{0} v_{1}^{\alpha_{21}} \\
u, v & \geq 0
\end{aligned} \text { in } \mathbb{R}^{N},\right.
$$

where $b_{0}, c_{0}>0, a_{0}, c_{1} \geq 0$. So (49) implies

$$
\left\{\begin{aligned}
-\Delta v_{1} & \geq b_{0} v_{2}^{\alpha_{12}} \\
-\Delta v_{2} & \geq c_{0} v_{1}^{\alpha_{21}} \\
u, v & \geq 0
\end{aligned} \quad \text { in } \quad \mathbb{R}^{N} .\right.
$$

We split the argument in two cases once again.

- If $\max \left\{\beta_{1}, \beta_{2}\right\} \geq N-2$, Theorem 2.2 applied to (50) gives $v_{1}=v_{2} \equiv 0$ in $\mathbb{R}^{N}$.

- Finally, if we have $\frac{N-2}{2}<\beta_{1}, \beta_{2}<N-2$, we apply Lemma 3.3 to (49), and conclude $v_{1}=v_{2} \equiv 0$ in $\mathbb{R}^{N}$, through the same argument as in the last part of the proof of Case A.

Proof in Case C. All that remains to consider are the cases when either $l_{3}$ meets $l_{1}$ at a point above $l_{4}$ or $l_{4}$ meets $l_{2}$ at a point below $l_{3}$. In both cases we take the couple $\left(\beta_{1}, \beta_{2}\right)$ to be this intersection point, that is, for instance in the first of these two situations

$$
\beta_{1}=\frac{2}{\alpha_{11}-1}, \quad \beta_{2}=\frac{2 \alpha_{11}}{\alpha_{12}\left(\alpha_{11}-1\right)} .
$$

Then, after passing to the limit in (14) we obtain a nontrivial bounded solution to the system

$$
\left\{\begin{array}{l}
-\Delta v_{1}=a_{0} v_{1}^{\alpha_{11}}+b_{0} v_{2}^{\alpha_{12}}+c_{1} v_{1}^{\gamma_{11}} v_{2}^{\gamma_{12}} \\
-\Delta v_{2}=0
\end{array}\right.
$$

in the whole space or in a half-space, with $v_{1}$ and $v_{2}$ vanishing on the boundary of the half space. Then Liouville's theorem applied to the second equation in (51) implies that $v_{2}$ is identically equal to a constant $c$. If $c=0$ (this is the only case if (51) is in a half-space, because of the boundary condition) we replace in the first equation in (51) and obtain a nontrivial solution to the Emden-Fowler equation (19) with $p=\alpha_{11}$, which is known not to have solutions for

$$
1<\alpha_{11}<\frac{N+2}{N-2}
$$


and this is a consequence of (8).

If $c>0$ the first equation in (51) implies that the inequality

$$
-\Delta v_{1} \geq b_{0} c^{\alpha_{12}}>0
$$

has a bounded solution in $\mathbb{R}^{N}$, which is well-known to be impossible.

\section{References}

[1] A.D. Alexandrov, A characteristic property of the spheres, Ann. Mat. Pura Appl. 58 (1962), 303-354, .

[2] C. Alves, D.G. de Figueiredo, Nonvariational elliptic systems, Discr. Cont. Dyn. Systems 8(2) (2002), 289-302.

[3] H. Amann, Fixed point equation and nonlinear eigenvalue problems in ordered Banach spaces, SIAM Review, 18 (1976), 620-709.

[4] H. Amann, On the number of solutions of nonlinear equations in ordered Banach spaces, J. Funct. Anal. 14 (1973), 349-381.

[5] C. Bandle, M. Essen, On positive solutions of Emden equations in cones. Arch. Rat. Mech. Anal. 112(4) (1990), 319-338.

[6] H. Berestycki, L. Caffarelli, L. Nirenberg, Further qualitative properties for elliptic equations in unbounded domains. Dedicated to Ennio De Giorgi. Ann. Scuola Norm. Sup. Pisa Cl. Sci. (4) 25(1-2) (1997), 69-94.

[7] H. Berestycki, L. Nirenberg, S.R.S. Varadhan, The principal eigenvalue and maximum principle for second order elliptic operators in general domains, Comm. Pure Appl. Math. 47(1) (1994), 47-92.

[8] I. Birindelli, E. Mitidieri, Liouville theorems for elliptic inequalities and inequations, Proc. Royal Soc. Edinburgh, 128A(1998), 1217-1247.

[9] H. Berestycki, L. Nirenberg, On the method of moving planes and the sliding method. Bull. Soc. Brazil Mat. Nova Ser. 22:1-37, (1991).

[10] J. Busca, R. Manasevich, A Liouville type theorem for Lane-Emden systems. Indiana Univ. Math. J. 51(1) (2002), 37-51.

[11] J. Busca, B. Sirakov, Symmetry results for semilinear elliptic systems in the whole space. J. Diff. Eq. 163(1) (2000), 41-56. 
[12] J. Busca, B. Sirakov, Harnack type estimates for nonlinear elliptic systems and applications, to appear in Ann. Inst. H. Poincare, Anal. Nonl.

[13] X. Cabre, On the Alexandrov-Bakelman-Pucci estimate and the reversed Hölder inequality for solutions of elliptic and parabolic equations, Comm. Pure and Appl. Math. 48 (1995), 539-570.

[14] X. Cabre, Topics in regularity and qualitative properties of solutions of nonlinear elliptic equations, Discr. Cont. Dyn. Syst. 8(2) (2002), 331359.

[15] W.X. Chen, C. Li, Classification of solutions of some nonlinear elliptic equations, Duke Math. J. 63 (1991), 615-623.

[16] Ph. Clement, D.G. de Figueiredo, E. Mitidieri, Positive solutions of semilinear elliptic systems. Comm. Part. Diff. Eq. 17 (1992), 923-940.

[17] E.N. Dancer, Some notes on the method of moving planes. Bull. Austral. Math. Soc. 46 (1992), 425-434.

[18] D.G. de Figueiredo, Monotonicity and symmetry of solutions of elliptic systems in general domains, NoDEA 1 (1994), 119-123.

[19] D.G. de Figueiredo, Semilinear elliptic systems. Nonl. Funct. Anal. Appl. Diff. Eq., World Sci. Publishing, River Edge, 122-152 (1998).

[20] D.G. de Figueiredo, P. Felmer, A Liouville-type theorem for elliptic systems. Ann. Sc. Norm. Sup. Pisa, 21 (1994), 387-397.

[21] D.G. de Figueiredo, P.-L. Lions, R. Nussbaum, A priori estimates and existence of positive solutions of semilinear elliptic equations, J. Math. Pures Appl. 61(1982), 41-63.

[22] B. Gidas, Symmetry properties and isolated singularities of positive solutions of nonlinear elliptic equations, Lect. Notes on Pure Appl. Math. 54 (1980), 255-273.

[23] B. Gidas, W.M. Ni, L. Nirenberg Symmetry and related properties via the maximum principle. Comm. Math. Phys. 6 (1981),883-901.

[24] B. Gidas, J. Spruck, A priori bounds for positive solutions of nonlinear elliptic equations. Comm. Part. Diff. Eq. (6) (1981), 883-901.

[25] D. Gilbarg, N.S. Trudinger, Elliptic Partial Differential EquaTIONS OF SECOND ORDER, 2nd edition, Revised Third Printing, Springer Verlag (1998). 
[26] J. Hulshof, R.C.A.M. van der Vorst, Differential systems with strongly indefinite variational structure. J. Funct. Anal. 114 (1993), 32-58.

[27] M.A. Krasnoselskii, Positive solutions of operator equations, P. Noordhoff, Groningen.

[28] Krylov, Nonlinear Elliptic And parabolic Equations of SECOND ORDER, Coll. Math. and its Appl. (1987).

[29] Laptev, G. G. Absence of global positive solutions of systems of semilinear elliptic inequalities in cones. (Russian) Izv. Ross. Akad. Nauk Ser. Mat. 64(6) (2000), 107-124; translation in Izv. Math. 64(6) (2000), $1197-1215$.

[30] E. Mitidieri, Non-existence of positive solutions of semilinear elliptic systems in $\mathbb{R}^{N}$. Quaderno Matematico 285 (1992).

[31] E. Mitidieri, Non-existence of positive solutions of semilinear elliptic systems in $\mathbb{R}^{N}$. Diff. Int. Eq. 9(3) (1996), 465-479.

[32] E. Mitidieri, S. Pohozaev, A priori estimates and the absence of solutions of nonlinear partial differential equations and inequalities. Tr. Mat. Inst. Steklova 234 (2001), 1-384.

[33] M.S. Montenegro, Criticalidade, superlinearidade e sublinearidade para sistemas elípticos semilineares, Tese de Doutoramento, Unicamp (1997).

[34] R. Nussbaum, Positive solutions of nonlinear elliptic boundary value problems, J. Math. Anal. Appl. 51 (1975), 461-482.

[35] J. Serrin A symmetry theorem in potential theory. Arch. Rat. Mech. Anal. 43 (1971), 304-318.

[36] J. Serrin, H. Zou, Non-existence of positive solutions of Lane-Emden systems. Diff. Int. Eq. 9(4) (1996), 635-653.

[37] B. Sirakov, Notions of sublinearity and superlinearity for nonvariational elliptic systems. In preparation.

[38] M.A.S. Souto, A priori estimates and and existence of positive solutions of nonlinear cooperative elliptic systems, Diff. Int. Eq. 8(5) (1995), 12451258.

[39] G. Sweers, Strong positivity in $C(\bar{\Omega})$ for elliptic systems, Math. Zeitschrift 209 (1992), 251-271. 
[40] H. Zou, A priori estimates for a semilinear elliptic system without variational structure and their application. Math. Ann. 323 (2002), 713-735. 